\title{
Unitarity, stability, and loops of unstable ghosts
}

\author{
John F. Donoghue $\odot^{*}$ \\ Department of Physics, University of Massachusetts Amherst, Amherst, Massachusetts 01003, USA \\ Gabriel Menezes $\circledast^{\dagger}$ \\ Department of Physics, University of Massachusetts Amherst, Amherst, Massachusetts 01003, USA \\ and Departamento de Física, Universidade Federal Rural do Rio de Janeiro, \\ 23897-000 Seropédica, RJ, Brazil
}

(Received 22 August 2019; published 12 November 2019)

\begin{abstract}
We present a new understanding of the unstable ghostlike resonance which appears in theories such as quadratic gravity and Lee-Wick type theories. Quantum corrections make this resonance unstable, such that it does not appear in the asymptotic spectrum. We prove that these theories are unitary to all orders. Unitarity is satisfied by the inclusion of only cuts from stable states in the unitarity sum. This removes the need to consider this as a ghost state in the unitarity sum. However, we often use a narrow-width approximation where we do include cuts through unstable states and ignore cuts through the stable decay products. If we do this with the unstable ghost resonance at one loop, we get the correct answer only by using a contour which was originally defined by Lee and Wick. The quantum effects also provide damping in both the Feynman and the retarded propagators, leading to stability under perturbations.
\end{abstract}

DOI: 10.1103/PhysRevD.100.105006

\section{INTRODUCTION}

Theories such as quadratic gravity [1-21] and Lee-Wick theories [22-30] have propagators which contain both quadratic and quartic momentum dependence. In addition to the pole at $q^{2}=0$, this combination will produce a high mass pole, via

$$
\frac{1}{q^{2}-\frac{q^{4}}{\mu^{2}}}=\frac{1}{q^{2}}-\frac{1}{q^{2}-\mu^{2}} .
$$

The negative sign for the second term implies that this pole is ghostlike, i.e., with negative norm. One therefore expects trouble with some combination of unitarity, stability, causality, etc. in such a theory. However, as Lee and Wick originally noted, interactions in such a theory make the heavy state unstable, with a width which can be calculated in perturbation theory. This feature is a crucial modification as it removes the ghost from the asymptotic spectrum. Past experience with Lee-Wick theories indicates that they can be stable and unitary, although causality does seem to be violated on microscopic scales of order the

\footnotetext{
*donoghue@physics.umass.edu gabrielmenezes@ufrrj.br
}

Published by the American Physical Society under the terms of the Creative Commons Attribution 4.0 International license. Further distribution of this work must maintain attribution to the author(s) and the published article's title, journal citation, and DOI. Funded by SCOAP ${ }^{3}$. width of the resonance $[22,26,28,31]$. In this paper, we describe further the unitarity and stability of such theories and come to an understanding of how unitarity is satisfied in the presence of unstable ghosts.

\section{A. Unitarity with normal resonances}

Unitarity describes the conservation of probability for the $S$ matrix. It states

$$
\langle f|T| i\rangle-\left\langle f\left|T^{\dagger}\right| i\right\rangle=i \sum_{j}\left\langle f\left|T^{\dagger}\right| j\right\rangle\langle j|T| i\rangle
$$

where we used the definition of the transfer matrix $T$, namely $S=\mathbf{1}+i T$. Here the associated states are the asymptotic single and multiparticle states of the theory. In processes that involve loop diagrams, the sum over real intermediate states can be accomplished by the Cutkosky cutting rules [32] which project out the on-shell states.

Procedurally, we often look first at the free-field theory to identify the free particles. Then when we include interactions, some of these particles become unstable and no longer appear as the asymptotic states of the theory. As far as the $S$ matrix is concerned, this is a significant change. The particles were originally needed in the Hilbert space for completeness, but then are no longer present in the interacting theory. The question then arises of how to treat such unstable particles in unitarity relations. Should one include them in the sums over states required for unitarity? 
The answer was provided by Veltman in 1963 [33]; see also [34-37]. He showed that unitarity is indeed satisfied by the inclusion of only the asymptotically stable states. Cuts are not to be taken through the unstable particles, and unstable particles are not to be included in unitarity sums.

However, there is a corollary which is useful in practice. In the narrow-width approximation, where the coupling to the decay products is taken to be very small, the offresonance production becomes small and only resonance production is important. As we demonstrate in Sec. VI, in this limit a cut taken through the unstable particle with its width set to zero reproduces the same result as a cut through the decay products.

This combination reinforces our intuition. The full calculation only requires the stable states, as unitarity demands. But when particles are nearly stable, we may approximate them as being stable in practical calculations.

\section{B. Unstable ghostlike resonances}

The ghostlike resonance in Lee-Wick type theories should also not be treated as an asymptotic state. In Sec. V, we will demonstrate how the Veltman's procedures can be employed for the calculation of cuts in diagrams involving such unstable ghostlike states and show that the usual cuts are obtained on the stable particles. Unitarity is satisfied for such cuts, and no cuts should be taken for the unstable ghost. There is no need for a modified contour in calculating the discontinuity, and the momentum integration runs along the real axis just above the cut.

However, a slight difference arises in defining the narrow-width approximation. While all the stable cuts are treated with the usual $i \epsilon$ prescription and are located below the integration contour, the unstable ghost carries a finite width and appears above the standard contour. If we wish to treat the unstable ghost as if it were a stable particle in the narrow-width approximation, we show that the correct answer is reproduced if and only if the contour is defined to pass above the location of the unstable pole. This is the origin of the Lee-Wick contour which was a somewhat puzzling feature of such theories. It is only invoked to reproduce the correct result of unitarity cuts through the stable particles.

\section{Why this matters}

In path-integral treatments, the stable states of a theory can generally be identified through the poles in the propagators. These then form the Hilbert space of the theory and are relevant for the unitarity relations. In the theories which we are discussing, it is relatively easy to identify that the ghostlike degrees of freedom are not part of the asymptotic spectrum.

In contrast, using canonical quantization we traditionally consider the free-field theory first. When trying to treat ghosts as regular free particles, we need to develop new quantization procedures. This task has occupied much of the literature on Lee-Wick type theories. Indeed, such procedures can be defined, although they appear somewhat unusual, and involve new concepts like indefinite metric or PT quantization [22,38-40]. One then debates whether to include such states in the unitarity sum or to define the path integration over such ghost variables.

While it is perhaps reassuring that canonical quantization is possible, it is an unnecessary step. Since these effects do not appear in the physical spectrum, we do not need to canonically quantize the ghosts. The path integral is over the physical field only, and the resonance appears as a calculable dynamical effect in the propagator. The resonance will have physical effects, but we do not need to describe its free-field quantization. Only stable states appear in the unitarity sum, and their cutting rules will be the standard ones. Our proof of unitarity in the presence of unstable resonances allows us to consider these theories further without discussing the canonical quantization of the unstable ghosts.

\section{GHOST RESONANCES}

The theories which we are studying have propagators of the form

$$
i D(q)=\frac{i}{q^{2}+i \epsilon-\frac{q^{4}}{M^{2}}+\Sigma(q)} .
$$

The pole at $q^{2}=0$ is the stable particle of the theory. The function $\Sigma(q)$ is the self-energy or vacuum polarization function. At high energies, it picks up an imaginary part due to coupling to light particles. At one-loop order, this typically has the form

$\Sigma(q)=-\frac{\gamma}{\pi} \log \left(\frac{-q^{2}-i \epsilon}{\mu^{2}}\right)=\left[-\frac{\gamma}{\pi} \log \left(\frac{\left|q^{2}\right|}{\mu^{2}}\right)+i \gamma \theta\left(q^{2}\right)\right]$

for some calculable quantity $\gamma$ with dimensions of mass squared. In general, $\gamma$ can be a function of the momentum and in quadratic gravity $\gamma \sim G q^{4}$. In our presentation here, for clarity we will neglect any masses of stable particles and the particles which appear in the vacuum polarization loop. It would be simple to restore any such masses.

The quartic momentum dependence is the novel feature in this propagator. It arises in theories which have four derivatives in the fundamental Lagrangian. We have chosen a particular sign for the quartic momentum dependence. The opposite sign would produce a tachyonic pole on the spacelike real axis, which appears to be fatal for the theory. With the sign shown in Eq. (3), there is a massive resonance for timelike values of $q^{2}$. Expanding near that resonance, we get the form 


$$
\left.i D(q)\right|_{q^{2} \sim m^{2}} \sim \frac{-i}{q^{2}-m^{2}-i \gamma} .
$$

The minus sign in the numerator tells us that this is a ghostlike resonance-it carries the opposite sign from the usual case. Equally important is that the width also comes with the opposite sign from normal. The resonance parameters are then $m_{R}^{2}=m^{2}+i \gamma$.

The point here is that the normal resonances and ghostlike resonances have the structure

$$
i D(q) \sim \frac{Z i}{q^{2}-m^{2}+i Z \gamma},
$$

with $Z=+1$ for a normal resonance and $Z=-1$ for the ghost resonance. The change of the two signs together is important. In particular, the imaginary part of the propagator is independent of $Z$,

$$
\operatorname{Im}[D(q)] \sim \frac{-\gamma}{\left(q^{2}-m^{2}\right)^{2}+\gamma^{2}} .
$$

We will show in Sec. II C that all viable (i.e., nontachyonic) theories with quartic momentum dependence fall into this class.

Note also that the stable particle and the resonance appear in the same propagator, Eq. (3), so that we need not be talking about two separate particles, but rather two features in the same propagator. However, because of the partial fraction relation, Eq. (1), it is equally possible to separate the propagator into the equivalent of two particles. It is also generally possible to do this by a field redefinition at the level of the Lagrangian.

\section{A. Quadratic gravity}

In quadratic gravity, the interesting case involves the spin-two propagator. The general Lagrangian has the form

$$
S_{\text {quad }}=\int d^{4} x \sqrt{-g}\left[\frac{2}{\kappa^{2}} R+\frac{1}{6 f_{0}^{2}} R^{2}-\frac{1}{\xi^{2}}\left(R_{\mu \nu} R^{\mu \nu}-\frac{1}{3} R^{2}\right)\right] .
$$

Here $\kappa^{2}=32 \pi G$. We have dropped the cosmological constant and will be expanding about Minkowski spacetime, $g_{\mu \nu}=\eta_{\mu \nu}+\kappa h_{\mu \nu}$. For more detail on this theory, see, for instance, $[1,7,8]$.

The spin-two propagator only depends on $\kappa$ and $\xi$. The vacuum polarization diagram adds a logarithmic term to the inverse propagator that is given by

$$
D_{2}^{-1}(q)=\cdots-\frac{\kappa^{2} q^{4} N_{\mathrm{eff}}}{640 \pi^{2}} \ln \left(\frac{-q^{2}-i \epsilon}{\mu^{2}}\right),
$$

where $N_{\text {eff }}$ is contribution of the light matter fields and normal gravitons, normalized to the contribution of massless vector fields. For timelike momenta, it generates an imaginary part

$$
\operatorname{Im}\left[D_{2}^{-1}(q)\right]=+\frac{\kappa^{2} q^{4} N_{\mathrm{eff}}}{640 \pi} \theta\left(q^{2}\right) .
$$

This generates the width for the ghost state. Our previous paper [8] on quadratic gravity has a detailed discussion of this feature.

The overall scalar part of the spin-two propagator is

$$
\begin{aligned}
D_{2}(q)= & \left\{q^{2}+i \epsilon-\frac{\kappa^{2} q^{4}}{2 \xi^{2}(\mu)}\right. \\
& \left.-\frac{\kappa^{2} q^{4} N_{\mathrm{eff}}}{640 \pi^{2}}\left[\ln \left(\frac{\left|q^{2}\right|}{\mu^{2}}\right)-i \pi \theta\left(q^{2}\right)\right]\right\}^{-1}
\end{aligned}
$$

for real values of the four momentum. This is shown for timelike momenta in Fig. 1. The unstable ghost resonance is visible. The peak occurs at momentum

$q_{r}^{2}=m_{r}^{2}=\frac{2 \xi^{2}(\mu)}{\kappa^{2}}+\frac{\xi^{2}(\mu) N_{\mathrm{eff}}}{320 \pi^{2} \kappa^{2}} \ln \left(\frac{\left|m_{r}^{2}\right|}{\mu^{2}}\right)=\frac{2 \xi^{2}\left(m_{r}\right)}{\kappa^{2}}$.

Evaluating the imaginary part at this momentum, one finds that the pole close to the real axis is located at

$$
M^{2}=m_{r}^{2}+i \gamma \quad \gamma=\xi^{2} m_{r}^{2} \frac{N_{\mathrm{eff}}}{320 \pi},
$$

valid for weak coupling. Weak coupling $\xi \ll 1$ implies that $m_{r}$ is smaller than the Planck mass, and that the width is narrow compared to the mass. The residue at the pole is -1 in weak coupling. For large values of $\xi$, the solutions for the mass, width, and residue must be found numerically.

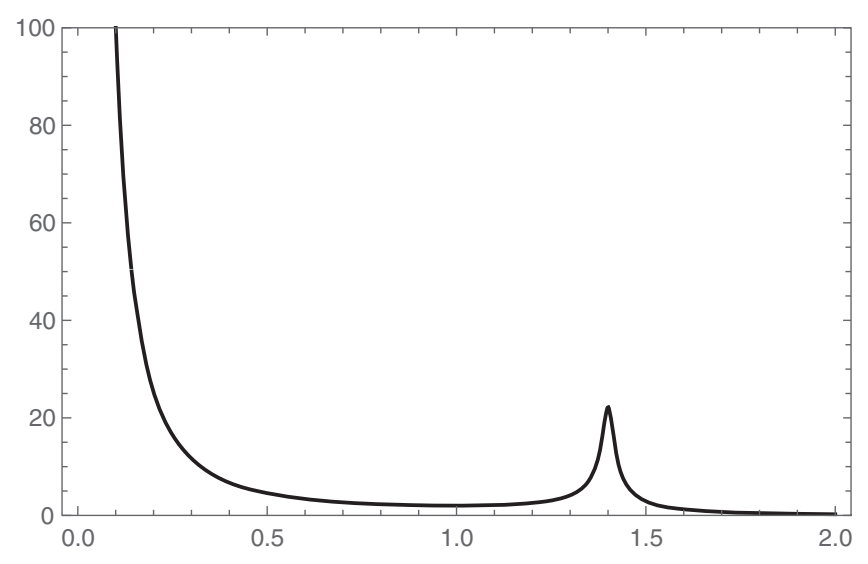

FIG. 1. The absolute value of the spin-two propagator for $\xi^{2}=1$, showing the high mass resonance. The $\mathrm{x}$-axis is the momentum $|q|$ in the timelike region, in units where $\kappa=1$. The imaginary parts have been calculated with loops of Standard Model particles and gravitons. 


\section{B. Lee-Wick QED}

Lee and Wick initiated a finite theory of QED, roughly by considering the Pauli-Villars regulator as a dynamical field. Because it enters with a minus sign, it also is ghostlike. The photon propagator in this case becomes

$$
i D_{F \mu \nu}\left(q^{2}\right)=-i g_{\mu \nu} D\left(q^{2}\right),
$$

with

$$
D\left(q^{2}\right)=\frac{1}{\left(q^{2}+i \epsilon\right)\left[1+\hat{\Pi}\left(q^{2}\right)-\frac{q^{2}}{\Lambda^{2}}\right]},
$$

with

$$
\hat{\Pi}\left(q^{2}\right)=q^{2} \frac{\alpha}{3 \pi} \int_{4 m_{f}^{2}}^{\infty} d s \frac{1}{s\left(s-q^{2}-i \epsilon\right)} \sqrt{1-\frac{4 m_{f}^{2}}{s}}\left(1+\frac{2 m_{f}^{2}}{s}\right) .
$$

Here $\hat{\Pi}\left(q^{2}\right)$ is the finite part of the vacuum polarization function for a fermion of mass $m_{f}$, written in a dispersion theory representation. At large $q^{2}$, the vacuum polarization becomes logarithmic. Near the resonance, it is more convenient to renormalize the theory using a running coupling. In this case, the result is

$$
\alpha D\left(q^{2}\right) \sim \frac{\alpha(\Lambda)}{q^{2}\left[1-\frac{\alpha}{3 \pi} \log \left(q^{2} / \Lambda^{2}\right)-\frac{q^{2}}{\Lambda^{2}}+i \frac{\alpha}{3}\right]}
$$

to first order in the coupling. The large logarithm has been absorbed into the coupling, and the remaining logarithmic running is weak near the pole.

This has the structure of our basic propagator, with $\gamma \sim \alpha \Lambda^{2} / 3$.

\section{Generic quartic propagators}

More generally, our work applies to other theories with four derivatives in the Lagrangian. Consider, for example, a scalar field $\phi$ which is coupled to other light fields $\chi$, with

$\mathcal{L}=\frac{1}{2}\left[\partial_{\mu} \phi \partial^{\mu} \phi-m^{2} \phi^{2}\right]-\frac{1}{2 M^{2}} \square \phi \square \phi-\frac{g}{2} \phi \sum_{i} \chi_{i}^{2}$

for $N$ light $\chi_{i}$ fields. The self-energy in this case proceeds through loops of the $\chi$ fields. It produces a residual selfenergy after mass renormalization

$$
\Sigma=-\frac{N g^{2}}{32 \pi^{2}}\left[\log \left(\left|q^{2}\right| / M^{2}\right)-i \pi \theta\left(q^{2}\right)\right] .
$$

This matches our general form with $\gamma=N g^{2} /(32 \pi)$ if we neglect the mass of the $\phi$ field.
A slightly better variant, which can serve as a good scalar proxy for quadratic gravity, is defined by the Lagrangian

$$
\mathcal{L}=\frac{1}{2} \partial_{\mu} \phi \partial^{\mu} \phi-\frac{\kappa^{2}}{4 \xi^{2}} \square \phi \square \phi-\frac{\kappa}{2}(\square \phi)\left(\phi^{2}+\sum_{i} \chi_{i}^{2}\right) .
$$

This variant does not need a mass for renormalization, because the interaction terms vanish on shell using the massless equations of motion. In addition, the momentumdependent interaction mimics the effect of the two derivatives of the gravitational interaction. Here, the residual self-energy is

$$
\Sigma=-\frac{\kappa^{2} q^{4}(N+1)}{32 \pi^{2}}\left[\log \left(\left|q^{2}\right| / M^{2}\right)-i \pi \theta q^{2}\right] .
$$

Indeed, the parameters have been chosen such that the resulting propagator is identically equal to the spin-two propagator of quadratic gravity, Eq. (11), with the identification of $N_{\text {eff }}=20(N+1)$. We will use this variation below in order to avoid the tensorial complications of real gravity.

We can take this last model and add an auxiliary field in order to accomplish at the Lagrangian level the factorization of the propagator that one sees using partial fraction relations. To do this, we introduce the auxiliary field $\eta$, using the Lagrangian

$$
\mathcal{L}=\frac{1}{2} \partial_{\mu} \phi \partial^{\mu} \phi-\square \phi \eta+\frac{\xi^{2}}{\kappa^{2}} \eta^{2}-\frac{\kappa}{2}(\square \phi)\left(\phi^{2}+\sum_{i} \chi_{i}^{2}\right) .
$$

Integrating out $\eta$ returns us to our original Lagrangian, Eq. (20). Now if we perform a field redefinition $\phi=h-\eta$, a little algebra turns this into

$$
\begin{aligned}
\mathcal{L}= & {\left[\frac{1}{2} \partial_{\mu} h \partial^{\mu} h-\frac{\kappa}{2} \square h \sum_{i} \chi_{i}^{2}\right] } \\
& -\left[\frac{1}{2}\left(\partial_{\mu} \eta \partial^{\mu} \eta-\frac{2 \xi^{2}}{\kappa^{2}} \eta^{2}\right)-\frac{\kappa}{2} \square \eta \sum_{i} \chi_{i}^{2}\right] \\
& -\frac{\kappa}{2}(\square h-\square \eta)(h-\eta)^{2} .
\end{aligned}
$$

Note in particular the overall minus sign in the second line.

From these examples, one is able to see that all theories with four derivative kinetic energies, $\sim \square \phi \square \phi$ will fall into the class of theories which we are discussing, as long as one avoids the tachyonic pole at spacelike momenta. The logic is as follows. Ordinary resonances arise when there is a coupling to the light states of the theory. The structure of the resonance propagator is 


$$
i D_{r}(q)=\frac{i}{q^{2}-m^{2}+\Sigma(q)}
$$

The imaginary part of the self-energy must be positive, that is

$$
\operatorname{Im}[\Sigma(q)]=\gamma(q) \theta\left(q^{2}\right)>0,
$$

such that the resonance mass $m^{2}-\operatorname{Re}[\Sigma]-i \operatorname{Im}[\Sigma]=$ $(M-i \Gamma / 2)^{2}$. Now if the Lagrangian is modified with a $\square^{2}$ term, the propagator gets modified to be

$$
i D(q)=\frac{i}{q^{2}-m^{2}+\Sigma(q)-q^{4} / \Lambda^{2}},
$$

where the sign on the new term has been chosen to avoid the tachyonic pole. If we set $\Lambda \rightarrow \infty$, we get a normal resonance where near the pole the propagator has the structure given in Eq. (6) with $Z=+1$. However, for large finite $\Lambda$, there is inevitably a high mass resonance, when $q^{2} \sim \Lambda^{2}$. For illustration, we can neglect $m^{2}$ and $\operatorname{Re}[\Sigma]$, and look at the structure near this resonance, such that

$$
\begin{aligned}
i D(q) & =\frac{i}{q^{2}-\frac{q^{4}}{\Lambda^{2}}+i \gamma(q)} \\
& =\frac{i}{\frac{q^{2}}{\Lambda^{2}}\left[\Lambda^{2}-q^{2}+i \gamma(q)\left(\Lambda^{2} / q^{2}\right)\right]} \\
& \sim \frac{-i}{q^{2}-\Lambda^{2}-i \gamma} .
\end{aligned}
$$

The residue at this pole is always negative-it is ghostlike. In addition, the sign of the width is always opposite from normal. That is, we find the correlated negative signs which we described in Eq. (6) with $Z=-1$. Indeed, for both finite $m$ and $\Lambda$, there will be resonances of both types contained in the same propagator. In both cases, the imaginary part of the self-energy arising from the coupling to stable states is the same, yet it manifests itself differently near the resonance because of the sign of the $q^{4}$ term.

\section{STABILITY AND ENERGY FLOW}

Because of the change in sign in front of the width in the denominator of the propagator, one might worry that there are exponentially growing modes. We show here that this is not the case. The stability of quadratic gravity has also been addressed using the equations of motion in curved backgrounds, although without including the decay width. The conclusion of [41] has been that the theory is stable in a curved background, at least for curvatures that are below the ghost mass. In [42], this was extended as at least metastability to curvatures beyond the scale of the ghost mass in the weakly coupled limit. Our treatment is somewhat different, remaining in Minkowski space but including the very important effect coming from the fact that the ghost decays.

An important feature also emerges from this analysis. The energy flow associated with the ghostlike terms in the propagator is different from the usual case. What we normally refer to as "positive energy" is seen to be propagating backward in time rather than the usual forward propagation. ${ }^{1}$ Indeed, the ghost propagator is the time reversed propagator of a normal resonance.

Let us study the coordinate space time dependence of the propagator by taking the Fourier transform, using the scalar part of the spin-two graviton propagator as our example

$$
i D_{2}(x)=\int \frac{d^{4} q}{(2 \pi)^{4}} e^{-i q \cdot x} i D_{2}(q) .
$$

A little algebra allows us to write

$$
D_{2}(q)=\left[\frac{1}{q^{2}}-\frac{1}{q^{2}-\mu^{2}(q)}\right]
$$

without any approximation, and

$\mu^{2}(q)=\frac{m_{r}^{4}\left[m_{r}^{2}+i \gamma \theta\left(q^{2}\right)+(\gamma / \pi) \log \left(\left|q^{2}\right| / m_{r}^{2}\right)\right]}{\left(m_{r}^{2}+\frac{\gamma}{\pi} \ln \left(\left|q^{2}\right| / m_{r}^{2}\right)\right)^{2}+\left(\gamma \theta\left(q^{2}\right)\right)^{2}}$.

In the weak coupling limit, $\gamma$ is a small number, so that we may approximate this by dropping the logarithmic term and keeping only the leading imaginary part. In this case, the propagator becomes

$$
D_{2}(q)=\left[\frac{1}{q^{2}}-\frac{1}{q^{2}-m_{r}^{2}-i \gamma \theta\left(q^{2}\right)}\right] .
$$

The propagator can be broken up into terms with forward propagation in time and backward,

$$
D_{2}(t, \vec{x})=\Theta(t) D_{\text {for }}(x)+\Theta(-t) D_{\text {back }}(x),
$$

with $x_{0}=t$. First consider $t>0$. In doing the $q_{0}$ integral, we need to close the contour in the lower half plane in order to have the contour at infinity give a vanishing contribution. There is the massless pole at

$$
q_{0}^{2}-\vec{q}^{2}+i \epsilon=0,
$$

which corresponds to $q_{0}= \pm\left(\omega_{q}-i \epsilon\right)$ with $\omega_{q}=|\vec{q}|$. There are massive poles at

$$
q_{0}^{2}-\vec{q}^{2}-m_{r}^{2}-i \gamma=0
$$

or

${ }^{1}$ In [31], we refer to these as "Merlin modes" in honor of the wizard in the Arthurian tales who ages backward in time. 


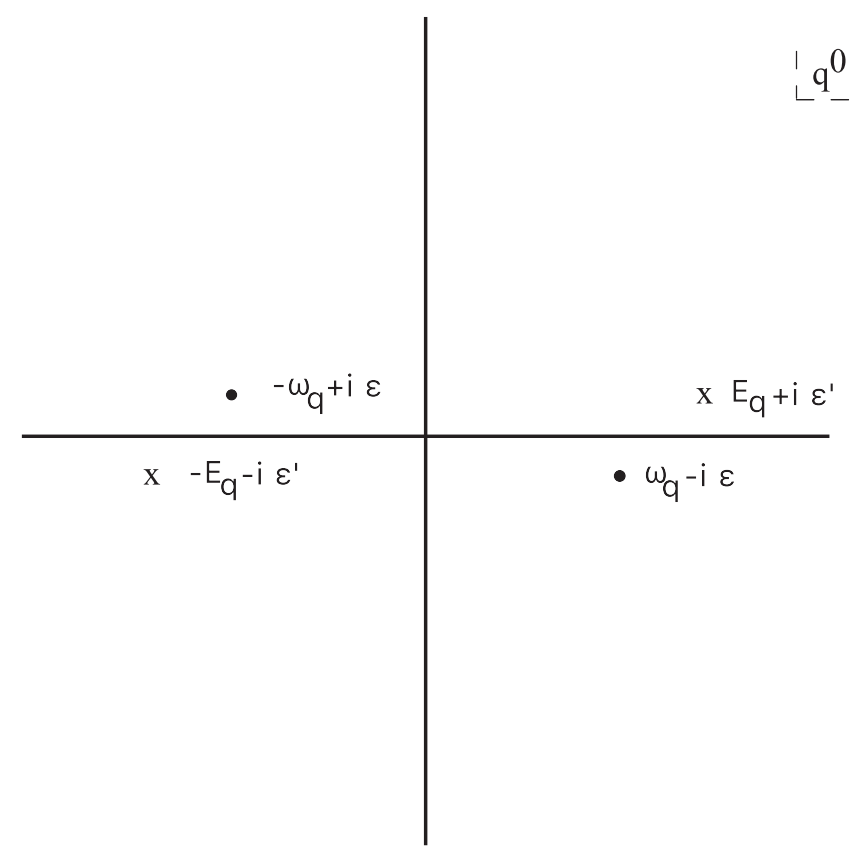

FIG. 2. The location of poles in the complex $q_{0}$ plane for the Feynman propagator.

$$
q_{0}= \pm \sqrt{E_{q}^{2}+i \gamma} \sim \pm\left[E_{q}+i \frac{\gamma}{2 E_{q}}\right]
$$

with $E_{q}=\sqrt{\vec{q}^{2}+m_{r}^{2}}$, which produces the pole structure shown in Fig. 2 . Performing the contour integral, we pick up the poles below the real axis. This yields the forward propagator

$D_{\text {for }}(t, \vec{x})=-i \int \frac{d^{3} q}{(2 \pi)^{3}}\left[\frac{e^{-i\left(\omega_{q} t-\vec{q} \cdot \vec{x}\right)}}{2 \omega_{q}}-\frac{e^{i\left(E_{q} t-\vec{q} \cdot \vec{x}\right)}}{2\left(E_{q}+i \frac{\gamma}{2 E_{q}}\right)} e^{-\frac{\gamma t}{2 E_{q}}}\right]$,

which shows the decaying exponential for the massive term, with the identification

$$
\gamma=m_{r} \Gamma
$$

Also we note that the energy flow of the two terms is different, with the massless pole carrying what we normally call positive energy, while the massive resonance carries negative energy. The term describing propagation backward in time is obtained for $t<0$ by closing in the upper half plane, with the result

$D_{\text {back }}(t, \vec{x})=-i \int \frac{d^{3} q}{(2 \pi)^{3}}\left[\frac{e^{i\left(\omega_{q} t-\vec{q} \cdot \vec{x}\right)}}{2 \omega_{q}}-\frac{e^{-i\left(E_{q} t-\vec{q} \cdot \vec{x}\right)}}{2\left(E_{q}+i \frac{\gamma}{2 E_{q}}\right)} e^{-\frac{\gamma|t|}{2 E_{q}}}\right]$.

Again we see exponential decay and the reversal of the energy flow between the two terms. We emphasize here that we are employing the usual contour for the propagator; we did not resort to any modified contour in order to obtain the above results.

One can also calculate the Green function with retarded and advanced boundary conditions. In the retarded case, the loop integrals going into the vacuum polarization need to be calculated using the in-in formalism. This has been done in Ref. [43]. The result is the same functional dependence, but with a different ie prescription. In particular, the logarithm becomes

$$
\begin{aligned}
\log & \left(-\left[\left(q_{0}+i \epsilon\right)^{2}-\vec{q}^{2}\right]\right) \\
\quad= & \log \left(-q^{2}-i \epsilon q_{0}\right) \\
\quad= & \log \left|q^{2}\right|-i \pi \theta\left(q^{2}\right)\left(\theta\left(q_{0}\right)-\theta\left(-q_{0}\right)\right) .
\end{aligned}
$$

This shifts the location of the poles to the positions indicated in Fig. 3.

For $t>0$, we pick up only the usual massless poles

$$
D_{\text {ret }}(t>0, \vec{x})=D_{\text {ret }}^{(0)}(t>0, \vec{x}) .
$$

However, here the unusual feature is that there is a contribution to the retarded Green function also for $t<0$,

$$
\begin{aligned}
D_{\text {ret }}(t<0, \vec{x}) \equiv & D_{\text {ret }}^{<}(t, \vec{x}) \\
= & i \int \frac{d^{3} q}{(2 \pi)^{3}}\left[\frac{e^{-i\left(E_{q} t-\vec{q} \cdot \vec{x}\right)}}{2\left(E_{q}+i \frac{\gamma}{2 E_{q}}\right)} e^{-\frac{\gamma|t|}{2 E_{q}}}\right. \\
& \left.-\frac{e^{i\left(E_{q} t-\vec{q} \cdot \vec{x}\right)}}{2\left(E_{q}-i \frac{\gamma}{2 E_{q}}\right)} e^{-\frac{\gamma|t|}{2 E_{q}}}\right] .
\end{aligned}
$$

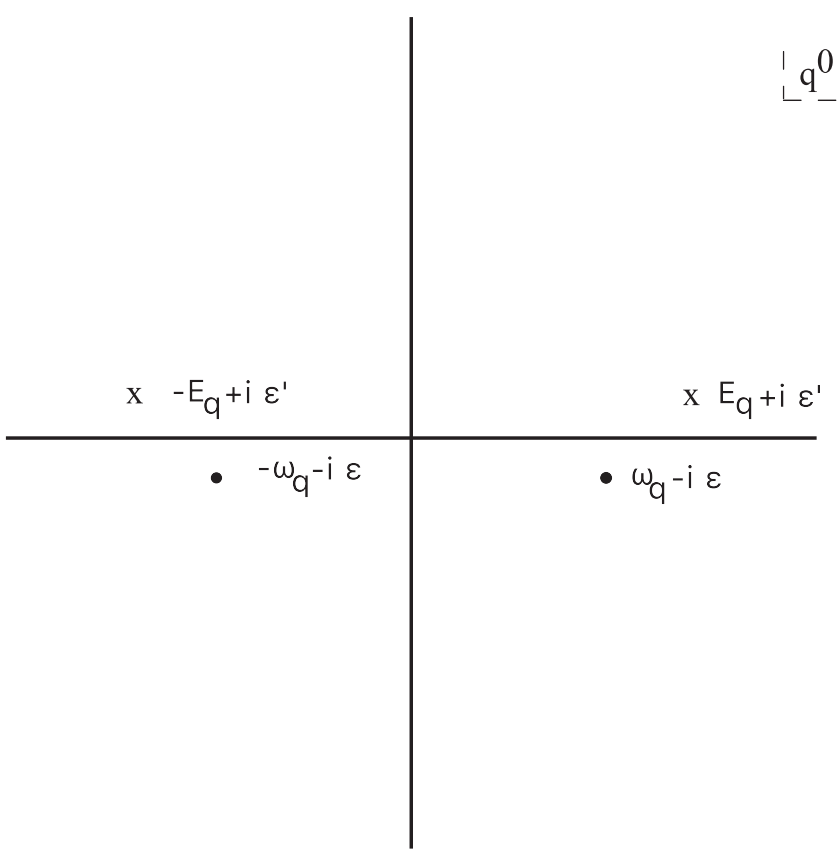

FIG. 3. The location of poles in the complex $q_{0}$ plane for the retarded Green function. 
This also contains decaying exponentials. If we choose to use this as a classical Green function giving the response to an external source,

$$
\begin{aligned}
h_{\mu \nu}(t, x)= & \int d^{3} x^{\prime}\left[\int_{-\infty}^{t} d t^{\prime} D_{\mathrm{ret}}^{(0)}\left(t-t^{\prime}, x-x^{\prime}\right)\right. \\
& \left.+\int_{t}^{\infty} d t^{\prime} D_{\mathrm{ret}}^{<}\left(t-t^{\prime}, x-x^{\prime}\right)\right] J_{\mu \nu}\left(t^{\prime}, x^{\prime}\right),
\end{aligned}
$$

it would correspond to the propagation of the effect backward in time. This is related to the microcausality violation on scales of order of the resonance width, known to be a feature of Lee-Wick type theories.

Finally, let us briefly comment on the formulation of our results in curved spacetime. One way to proceed is to consider the usual local momentum representation of the Feynman propagator, which employs Riemann normal coordinates [44,45]. But the straightforward expansion of the action is not enough. Here the loop diagram is also an essential ingredient since it generates the width for the ghost state. The associated expansion in curvature of this result can be relatively complicated. Indeed, one of us has calculated the full one-loop diagram for the photon propagator [46,47]. Such considerations can be carried out for Lee-Wick type theories, but that would take us a bit far from the scope of the present work. We plan to return to this topic in a future publication.

\section{CUTS WITH STABLE AND UNSTABLE PARTICLES}

Unitarity is realized by including only the stable particles in the unitarity sum. Because the all-orders proof of this including the unstable ghost, given in the next section using the methods of Veltman, is somewhat contrived and nonintuitive, we here give some simple examples to explore how this works. These examples also are useful in the discussion of the narrow-width approximation, which follows in Sec. VI.

In calculating the discontinuity of a process or a Feynman diagram, one cuts the diagram(s) in all possible ways. For the propagators in the cut, one replaces the propagator by an on-shell delta function

$$
\frac{i}{q^{2}-m^{2}+i \epsilon} \rightarrow 2 \pi \delta\left(q^{2}-m^{2}\right) \theta\left(q_{0}\right)
$$

One also replaces all propagators on the far right-hand side of the cut (typically represented by a shaded region) by their complex conjugate. ${ }^{2}$ This amounts to replacing $+i \epsilon$ by $-i \epsilon$,

\footnotetext{
${ }^{2}$ Not all textbooks include this latter rule, although it is important in multiloop diagrams.
}

$$
\frac{i}{q^{2}-m^{2}+i \epsilon} \rightarrow \frac{-i}{q^{2}-m^{2}-i \epsilon}
$$

for these propagators.

In Ref. [8], we discussed the scattering in the spin-two partial wave in quadratic gravity and found it to be unitary. Specifically, we found

$$
T_{2}(s)=-\frac{N_{\mathrm{eff}} s}{640 \pi} \bar{D}_{2}(s) .
$$

This is normalized such that in order to satisfy elastic unitarity, we must have $\operatorname{Im} T_{2}=\left|T_{2}\right|^{2}$. This is satisfied when the amplitude has the form

$$
T_{2}(s)=\frac{A(s)}{f(s)-i A(s)}=\frac{A(s)[f(s)+i A(s)]}{f^{2}(s)+A^{2}(s)}
$$

for any real functions $f(s), A(s)$. This relation is satisfied with

$$
A(s)=-\frac{N_{\mathrm{eff}} s}{640 \pi} .
$$

Because this example is essentially just scattering through the spin-two propagator, we start with the calculation of the discontinuity in propagators.

\section{A. Two-particle cut}

For simplicity, we here study a scalar propagator, using the scalar proxy for quadratic gravity, Eq. (20). The vacuum polarization enters the propagator through the bubble sum. The cuts available in the bubble sum are shown in Fig. 4. The calculation of the basic cut is straightforward,

$$
\begin{aligned}
\operatorname{Disc}_{2} \Sigma(q)= & \frac{\kappa^{2} q^{4}(N+1)}{2} \int \frac{d^{4} k}{(2 \pi)^{4}} 2 \pi \delta\left(k^{2}\right) \theta\left(k_{0}\right) \\
& \times 2 \pi \delta\left((q-k)^{2}\right) \theta\left((q-k)_{0}\right) .
\end{aligned}
$$

The result is

$$
\operatorname{Disc}_{2} \Sigma\left(q^{2}\right)=\frac{(N+1) \kappa^{2} q^{4}}{32 \pi}
$$

Here the $(N+1)$ factor counts the number of massless states that the resonance can decay into.

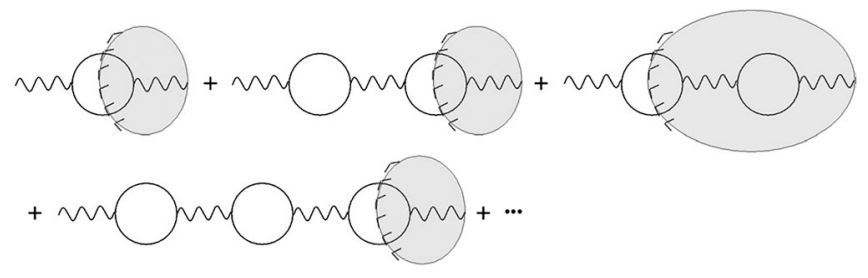

FIG. 4. The two-particle cuts in the propagator. 
We can also repackage the discontinuity in terms of the two-body decay width. This can be done by inserting a factor of

$$
\int \frac{d^{4} p}{(2 \pi)^{4}}(2 \pi)^{4} \delta^{4}(q-k-p)=1
$$

and identifying the matrix element $\mathcal{M}_{2}=\kappa q^{2}$, to write

$$
\operatorname{Disc}_{2} \Sigma(q)=\frac{(N+1)}{2} \int \frac{d^{4} k}{(2 \pi)^{4}} \frac{d^{4} p}{(2 \pi)^{4}}(2 \pi)^{4} \delta^{4}(q-k-p)\left|\mathcal{M}_{2}\right|^{2} 2 \pi \delta\left(k^{2}\right) \theta\left(k_{0}\right) 2 \pi \delta\left((q-k)^{2}\right) \theta\left((q-k)_{0}\right) .
$$

The on-shell delta functions can be used to do the integration over the energy variables, leaving

$$
\operatorname{Disc}_{2} \Sigma(q)=\frac{(N+1)}{2} \int \frac{d^{3} k}{(2 \pi)^{3} 2 \omega_{k}} \frac{d^{3} p}{(2 \pi)^{3} 2 \omega_{p}}(2 \pi)^{4} \delta^{4}(q-k-p)\left|\mathcal{M}_{2}\right|^{2}
$$

This latter relation can be recognized as the two-body decay width at the overall center of mass momentum $q$, up to an initial normalization factor of $1 / 2 q$, resulting in

$$
\operatorname{Disc}_{2} \Sigma(q)=2 q \Gamma_{2}(q)
$$

which is another statement of the unitarity relation.

When we take cuts in diagrams with multiple bubbles, the bubbles on each side of the cut can be summed to yield the dressed propagator. Recall that on shaded regions, the bubbles are calculated with a $-i \epsilon$ prescription, which results in the complex conjugate of the usual bubble diagram. Performing this bubble sum, the result is

$\operatorname{Disc} D(q)=D(q) 2 q \Gamma_{2}(q) D^{*}(q)=-2 \operatorname{Im}[D(q)]$, with

$$
D(q)=\frac{1}{q^{2}-\frac{\kappa^{2} q^{4}}{2 \xi^{2}(\mu)}-\frac{(N+1) \kappa^{2} q^{4}}{32 \pi^{2}}\left[\log \left(q^{2} / \mu^{2}\right)-i \pi\right]},
$$

which is the expected discontinuity relation for the propagator.

\section{B. Three-particle cut}

More interesting is the three-particle cut, such as that shown in Fig. 5. Here the basic cut is in a two loop integral.

$$
\begin{aligned}
\operatorname{Disc}_{3} \Sigma(q)= & \kappa^{2} q^{4} \int \frac{d^{4} k_{1}}{(2 \pi)^{4}} \frac{d^{4} k_{2}}{(2 \pi)^{4}} \frac{1}{\left(q-k_{1}\right)^{2}-\frac{\kappa^{2}\left(q-k_{1}\right)^{4}}{2 \xi^{2}}} \frac{(N+1) \kappa^{2}\left(q-k_{1}\right)^{2}}{2} \\
& \times 2 \pi \delta\left(k_{1}^{2}\right) \theta\left(k_{10}\right) 2 \pi \delta\left(k_{2}^{2}\right) \theta\left(k_{20}\right) 2 \pi \delta\left(\left(q-k_{1}-k_{2}\right)^{2}\right) \theta\left(\left(q-k_{1}-k_{2}\right)_{0}\right) \frac{1}{\left(q-k_{1}\right)^{2}-\frac{\kappa^{2}\left(q-k_{1}\right)^{4}}{2 \xi^{2}}} .
\end{aligned}
$$

Here again there are bubble diagrams on each side of the cut, and these can be summed to yield the dressed propagator, with the propagators on the shaded regions being complex conjugated. We can package this more compactly, using the matrix element

$$
\mathcal{M}_{3}=\kappa q^{2} \kappa\left(q-k_{1}\right)^{2} D\left(q-k_{1}\right)
$$

with the propagator $D$ being given by Eq. (55). Using this, we have

$$
\operatorname{Disc}_{3} \Sigma(q)=\frac{N+1}{2} \int \frac{d^{4} k_{1}}{(2 \pi)^{4}} \frac{d^{4} k_{2}}{(2 \pi)^{4}}\left|\mathcal{M}_{3}\right|^{2} 2 \pi \delta\left(k_{1}^{2}\right) \theta\left(k_{10}\right) 2 \pi \delta\left(k_{2}^{2}\right) \theta\left(k_{20}\right) 2 \pi \delta\left(\left(q-k_{1}-k_{2}\right)^{2}\right) \theta\left(\left(q-k_{1}-k_{2}\right)_{0}\right) .
$$

We can add an extra integration and an extra delta function by using momentum conservation to write $k_{3}=q-k_{1}-k_{2}$, such that 
$\operatorname{Disc}_{3} \Sigma(q)=\frac{N+1}{2} \int \frac{d^{4} k_{1}}{(2 \pi)^{4}} \frac{d^{4} k_{2}}{(2 \pi)^{4}} \frac{d^{4} k_{3}}{(2 \pi)^{4}}(2 \pi)^{4} \delta^{4}\left(q-k_{1}-k_{2}-k_{3}\right)\left|\mathcal{M}_{3}\right|^{2} 2 \pi \delta\left(k_{1}^{2}\right) \theta\left(k_{10}\right) 2 \pi \delta\left(k_{2}^{2}\right) \theta\left(k_{20}\right) 2 \pi \delta\left(\left(k_{3}\right)^{2}\right) \theta\left(\left(k_{30}\right)\right.$.

Now the on-shell delta functions can be performed by doing the energy integral in each case,

$$
\operatorname{Disc}_{3} \Sigma(q)=\frac{N+1}{2} \int \frac{d^{3} k_{1}}{(2 \pi)^{3} 2 \omega_{1}} \frac{d^{3} k_{2}}{(2 \pi)^{3} 2 \omega_{2}} \frac{d^{3} k_{3}}{(2 \pi)^{3} 2 \omega_{3}}(2 \pi)^{4} \delta^{4}\left(q-k_{1}-k_{2}-k_{3}\right)\left|\mathcal{M}_{3}\right|^{2} .
$$

At this stage, we recognize the formula for the three body decay width $\Gamma_{3}$, such that

$$
\operatorname{Disc}_{3} \Sigma(q)=2 q \Gamma_{3}(q)
$$

which is the desired unitarity relation.

Besides the cut which we just calculated there is another one which does not involve the ghost in any way. It is shown in Fig. 6. It is simple to evaluate and just gives an extra term in the matrix element $\mathcal{M}$.

\section{Heuristic proof of unitarity}

The above calculations allow us to get a feel for why such theories satisfy unitarity even beyond these simple examples. We have seen that unitarity works with the stable particles as external states in the unitarity sum. The ghost resonance does not occur as an external state. This is the same pattern as normal resonances, and the above calculation could be performed identically for normal resonances.

We also know from the analysis of Sec. II C that normal resonances and ghost resonances can be described in the same propagator using the coupling to the stable states described by the same $\Sigma(q)$.

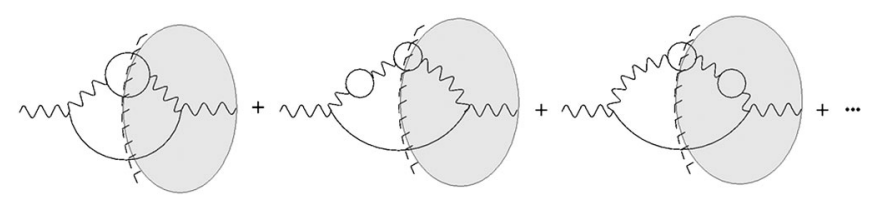

FIG. 5. The three-particle cuts in the propagator.

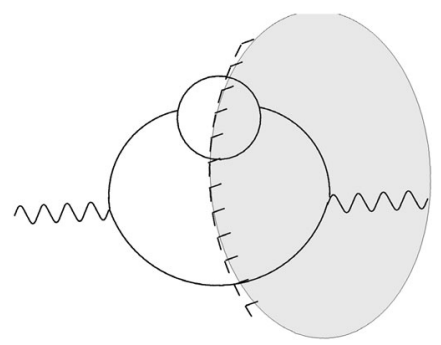

FIG. 6. Another three-particle cut in the self-energy function.
Finally, we know from Veltman's work that normal resonances satisfy unitarity to all orders. It then follows that any discontinuity calculated with normal resonances in the intermediate states, can be converted into a discontinuity with ghost resonances by using Eq. (26). If the normal resonance satisfies the unitarity relation, the ghost resonance will also.

The above heuristic argument appears to be correct in direct calculations. Comparison with the formal proof of Veltman however superficially appears to indicate some differences between the two cases. This has to do with the differences with energy flow in the different parts of the propagators, a feature that we have explored in Sec. III. We will see that this difference can be accounted for and unitarity holds. Other perspectives on unitarity with ghosts are given in $[2,5,6,8,14,18]$. We turn to the formal proof next.

\section{PROOF OF UNITARITY WITH UNSTABLE GHOSTS}

In this section, we give a proof that theories with unstable ghosts are unitary to all orders. The demonstration follows Veltman's argument [33]. For such purposes, we will derive the so-called cutting equation which implies unitarity, as we will discuss. The cutting equation contains terms associated with the imaginary part of propagators, given by the cut propagators. Essentially, the cutting equation is to be identified with the generalized optical theorem associated with an arbitrary process $a \rightarrow b$,

$$
\begin{aligned}
& i \mathcal{M}(a \rightarrow b)-i \mathcal{M}^{*}(b \rightarrow a) \\
& \quad=-\sum_{f} \int d \Pi_{f} \mathcal{M}^{*}(b \rightarrow f) \mathcal{M}(a \rightarrow f)(2 \pi)^{4} \delta^{4}(a-f),
\end{aligned}
$$

which is just Eq. (2) with the definition $\langle f|T| i\rangle=$ $(2 \pi)^{4} \delta^{4}\left(p_{i}-p_{f}\right) \mathcal{M}(i \rightarrow f)$. The quantity $d \Pi_{f}$ is the Lorentz-invariant phase space [48] and the sum runs over all possible sets $f$ of intermediate states (single and multiparticle states), and there is an overall delta function assuring energy-momentum conservation. In the above expression, the $\mathcal{M}$ 's are the invariant scattering matrix 
elements which are the nontrivial part of the $S$ matrix. On the other hand, the usual physical interpretation of the Lehmann-Symanzik-Zimmermann (LSZ) formula is that the $S$ matrix projects out one-particle asymptotic states from the time-ordered product of fields [48]. Since the spectral function contains contributions from multiparticle intermediate states with a continuous mass spectrum, this implies that the contribution coming from the cut propagators in the cutting equation should only contain the terms associated with the one-particle states. This also implies that the contributions coming from unstable particles are expected to be absent in Eq. (62). This is what we wish to prove here for models with unstable ghosts.

\section{A. Lehmann representations}

We begin by establishing our assumptions and some basic definitions. We assume that an expression for the propagator in terms of a Lehmann representation $[49,50]$ is available, that is

$$
G\left(p^{2}\right)=\frac{1}{\pi} \int_{0}^{\infty} d s \frac{\sigma(s)}{p^{2}-s+i \epsilon}
$$

for stable particles, and

$$
\tilde{G}\left(p^{2}\right)=\frac{1}{\pi} \int_{0}^{\infty} d s \frac{\tilde{\rho}(s)}{p^{2}-s+i \epsilon}
$$

for normal unstable particles. As remarked above, we neglect any masses of stable particles.

For stable particles, there should be an isolated deltafunction contribution due to one-particle states,

$$
\sigma(s)=\mathcal{Z} \pi \delta(s)+\rho(s) .
$$

On the other hand, for unstable particles, the "one-particle pole" is right on the branch cut; interactions essentially move the "pole" off the branch cut, but at the cost of introducing an imaginary part into it. In this case, one does not verify the presence of an asymptotic state according to the LSZ reduction formula and it is in this sense that such a particle is said to be unstable: There cannot be a isolated delta-function contribution to the spectral function since there are no one-particle states in the usual sense. In this case, in the narrow-width approximation, the spectral function has a Breit-Wigner shape,

$$
\tilde{\rho}(s) \approx \frac{M \Gamma}{\left(s-M^{2}\right)^{2}+M^{2} \Gamma^{2}},
$$

where $\Gamma$ is the width of the resonance. For the normal unstable particle, the Lehmann representation has $\tilde{\rho}(s)>0$ and $\epsilon>0$.

For theories with unstable ghosts, such as Lee-Wick QED and our scalar proxy for quadratic gravity given by
Eq. (20), as discussed above, the positive energy flow associated with the ghost propagates backward in time. Moreover, the residue at the pole associated with the unstable ghost is always negative and the sign of the width is always opposite from normal resonances. For such reasons, for the ghost field we propose the following Lehmann representation:

$$
\tilde{G}_{\mathrm{GH}}\left(p^{2}\right)=-\frac{1}{\pi} \int_{0}^{\infty} d s \frac{\tilde{\rho}(s)}{p^{2}-s-i \epsilon} .
$$

This amounts to considering a "anti time-ordered product" in the definition of the propagator. This full spectral representation for the LW propagator can be easily obtained from the modified Lehmann representation presented in Refs. [28,51]—one just needs to employ a spectral function with a Breit-Wigner shape (in the narrow-width approximation) for both complex-conjugate poles.

In setting up the above Lehmann representations, we are clearly resorting to the partial fraction relation given by Eq. (1) for the case of the unstable ghost. We use this equivalent form for convenience and also for clarity of presentation. In any case, it is important to bear in mind that the stable particle and the ghostlike resonance are both parts of the same propagator, so the consideration of both entities as two separate particles cannot be pivotal to the procedure undertaken here. As such, all the arguments and results obtained in this section can be generalized for a single propagator such as the one given by Eq. (26).

In the Lehmann representation for the stable particle presented above, $\mathcal{Z}=1$ for the free theory. In such a case, field operators can only create a single particle from the vacuum. Hence, the contributions from multiparticle intermediate states, encoded in the spectral functions $\rho(s)$ and $\tilde{\rho}(s)$, are only present in the full interacting theory. In other words, the information about interactions are contained in $\mathcal{Z}$ as well as in the spectral functions. This implies that the Lehmann representation for unstable particles will never contain a "free-field" contribution. We will return to this topic in due course.

\section{B. Cut propagators}

In what follows, an important ingredient in our discussion of unitarity is the decomposition of the propagator into terms with forward and backward propagation in time. For the stable particle, one gets

$$
\begin{aligned}
i G\left(x-x^{\prime}\right)= & \Theta\left(x_{0}-x_{0}^{\prime}\right) G^{+}\left(x-x^{\prime}\right) \\
& +\Theta\left(-x_{0}+x_{0}^{\prime}\right) G^{-}\left(x-x^{\prime}\right),
\end{aligned}
$$

where, using the spectral representation

$$
G^{ \pm}\left(x-x^{\prime}\right)=\int \frac{d^{4} p}{(2 \pi)^{3}} e^{-i p \cdot\left(x-x^{\prime}\right)} \theta\left( \pm p_{0}\right) \frac{\sigma\left(p^{2}\right)}{\pi} .
$$


The Green's functions $G^{ \pm}$are also known as cut propagators. Using that

$$
\begin{aligned}
& i G^{ \pm}\left(x-x^{\prime} ; m^{2}\right) \\
& \quad= \pm 2 \pi \int \frac{d^{4} p}{(2 \pi)^{4}} e^{-i p \cdot\left(x-x^{\prime}\right)} \theta\left( \pm p_{0}\right) \delta\left(p^{2}-m^{2}\right),
\end{aligned}
$$

one can also write

$$
G^{ \pm}\left(x-x^{\prime}\right)= \pm \frac{1}{\pi} \int_{0}^{\infty} d s \sigma(s) i G^{ \pm}\left(x-x^{\prime} ; s\right) .
$$

Hence, in momentum space

$$
G^{ \pm}\left(p^{2}\right)=2 \pi \theta\left( \pm p_{0}\right) \int_{0}^{\infty} d s \delta\left(p^{2}-s\right) \frac{\sigma(s)}{\pi} .
$$

For the normal unstable particle, one finds a similar representation in terms of cut propagators, namely

$$
\begin{aligned}
i \tilde{G}\left(x-x^{\prime}\right)= & \Theta\left(x_{0}-x_{0}^{\prime}\right) \tilde{G}^{+}\left(x-x^{\prime}\right) \\
& +\Theta\left(-x_{0}+x_{0}^{\prime}\right) \tilde{G}^{-}\left(x-x^{\prime}\right),
\end{aligned}
$$

where

$$
\tilde{G}^{ \pm}\left(x-x^{\prime}\right)= \pm \frac{1}{\pi} \int_{0}^{\infty} d s \tilde{\rho}(s) i G^{ \pm}\left(x-x^{\prime} ; s\right) .
$$

In momentum space,

$$
\tilde{G}^{ \pm}\left(p^{2}\right)=2 \pi \theta\left( \pm p_{0}\right) \int_{0}^{\infty} d s \delta\left(p^{2}-s\right) \frac{\tilde{\rho}(s)}{\pi} .
$$

The case of unstable ghostlike resonance is more subtle since here energy flow is backward, among other features discussed above. Following the prescription proposed above, we write

$$
\begin{aligned}
i \tilde{G}_{\mathrm{GH}}\left(x-x^{\prime}\right)= & \Theta\left(x_{0}-x_{0}^{\prime}\right) \tilde{G}_{\mathrm{GH}}^{-}\left(x-x^{\prime}\right) \\
& +\Theta\left(-x_{0}+x_{0}^{\prime}\right) \tilde{G}_{\mathrm{GH}}^{+}\left(x-x^{\prime}\right),
\end{aligned}
$$

where

$$
\tilde{G}_{\mathrm{GH}}^{ \pm}\left(x-x^{\prime}\right)=\mp \frac{1}{\pi} \int_{0}^{\infty} d s \tilde{\rho}(s) i G^{ \pm}\left(x-x^{\prime} ; s\right) .
$$

In momentum space,

$$
\tilde{G}_{\mathrm{GH}}^{ \pm}\left(p^{2}\right)=-2 \pi \theta\left( \pm p_{0}\right) \int_{0}^{\infty} d s \delta\left(p^{2}-s\right) \frac{\tilde{\rho}(s)}{\pi} .
$$

For all cases above, in view of the reality of the spectral functions, one has $G^{ \pm}=\left(G^{\mp}\right)^{*}$. Hence, one finds that

$$
\begin{aligned}
-i G^{*}\left(x-x^{\prime}\right)= & \Theta\left(x_{0}-x_{0}^{\prime}\right) G^{-}\left(x-x^{\prime}\right) \\
& +\Theta\left(-x_{0}+x_{0}^{\prime}\right) G^{+}\left(x-x^{\prime}\right) \\
-i \tilde{G}^{*}\left(x-x^{\prime}\right)= & \Theta\left(x_{0}-x_{0}^{\prime}\right) \tilde{G}^{-}\left(x-x^{\prime}\right) \\
& +\Theta\left(-x_{0}+x_{0}^{\prime}\right) \tilde{G}^{+}\left(x-x^{\prime}\right) \\
-i \tilde{G}_{\mathrm{GH}}^{*}\left(x-x^{\prime}\right)= & \Theta\left(x_{0}-x_{0}^{\prime}\right) \tilde{G}_{\mathrm{GH}}^{+}\left(x-x^{\prime}\right) \\
& +\Theta\left(-x_{0}+x_{0}^{\prime}\right) \tilde{G}_{\mathrm{GH}}^{-}\left(x-x^{\prime}\right) .
\end{aligned}
$$

\section{Largest time equation}

Let us construct the so-called largest time equation. Consider an arbitrary connected diagram with $n$ vertices. All such vertices carry a spacetime coordinate $x_{i}$. We represent the diagram by the function $F\left(x_{1}, x_{2}, \ldots, x_{n}\right)$. As usual, each line connecting any two vertices $x_{i}$ and $x_{j}$ in $F$ is associated with a propagator. Suppose there is a coordinate $x_{m}$ associated with a given stable particle with the largest time component. Then clearly $i G\left(x_{m}-x_{j}\right)=$ $G^{+}\left(x_{m}-x_{j}\right)$ and $i G\left(x_{k}-x_{m}\right)=G^{-}\left(x_{k}-x_{m}\right)$ for all $x_{k}$, $x_{j} \in F$. Now consider the operation of circling the vertices of the diagram. Feynman rules can be introduced in order to take into account such an action.

(i) A line joining two uncircled (circled) vertices is associated with $G\left(G^{*}\right)$.

(ii) A line connecting a circled (uncircled) vertex $x_{k}$ to an uncircled (circled) vertex $x_{i}$ is to be associated with $G^{+}\left(x_{k}-x_{i}\right)\left(G^{-}\left(x_{k}-x_{i}\right)\right)$.

(iii) For any circled vertex, one associates an overall minus sign.

For a thorough discussion, see Ref. [34]. The first two rules are summarized in Fig. 7. To understand the third rule,

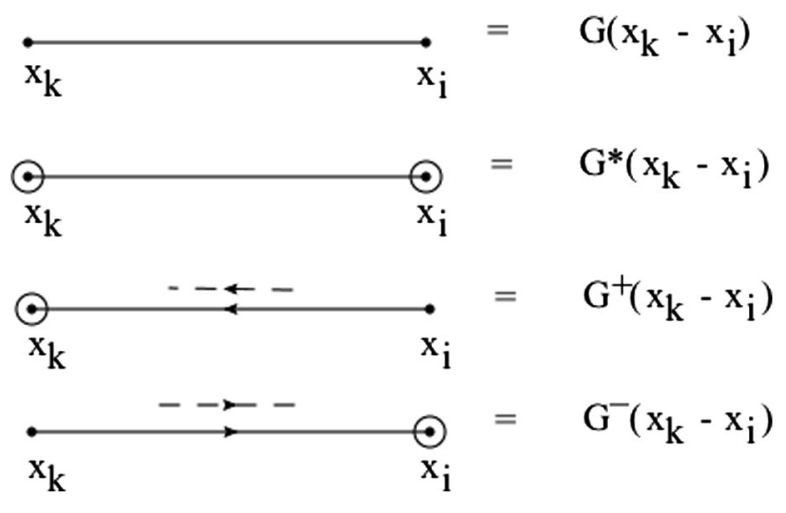

FIG. 7. Circling rules. The last two expressions are valid for stable particles and normal unstable particles. The arrow in the thick line connecting vertices represents the (positive) energy flow, whereas the arrow in the dashed line represents the flow of time. 


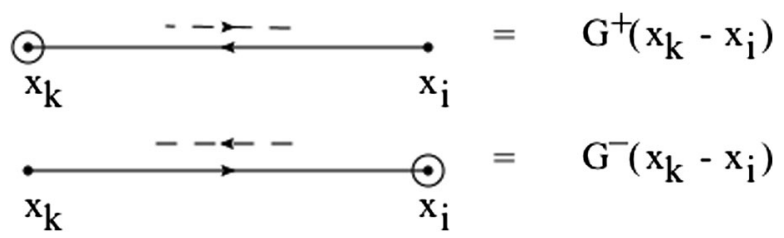

FIG. 8. Circling rules for the unstable ghost. Observe that energy flows in the opposite direction of the flow of time, in accordance with the description in the text.

consider, for instance, a generic diagram given by the expression

$$
F\left(x_{1}, x_{2}, x_{3}\right)=(i g)^{3} G\left(x_{1}-x_{2}\right) G\left(x_{2}-x_{3}\right) G\left(x_{3}-x_{1}\right) .
$$

If we circle the vertices corresponding to $x_{2}$ and $x_{3}$, then the circling rules tell us that

$$
\begin{aligned}
F\left(x_{1}, \underline{x}_{2}, \underline{x}_{3}\right)= & (-i g)^{2}(i g) G^{-}\left(x_{1}-x_{2}\right) \\
& \times G^{*}\left(x_{2}-x_{3}\right) G^{+}\left(x_{3}-x_{1}\right),
\end{aligned}
$$

where the coordinates related to the circled vertices were underlined. The $(-i g)^{2}$ in the above equation is the consequence of the usage of the third rule. Observe that one of the most important features of such rules is that energy flows from uncircled to circled vertices due to the presence of the theta function $\theta\left( \pm p_{0}\right)$ in the definition of $G^{ \pm}\left(x-x^{\prime} ; m^{2}\right)$; see Fig. 7. This feature is valid for all three cases, stable particle, normal unstable particles, and unstable ghosts. The crucial difference is that for the latter the time flows in the opposite direction of the (positive) energy flow; see Fig. 8.

An immediate consequence of the above circling rules is that, if one considers an arbitrary circling of $F\left(x_{1}\right.$, $\left.x_{2}, \ldots, x_{n}\right)$ and add to it the same resulting diagram but with the opposite circling of $x_{m}$, then the result is zero. In particular, if one considers all the $2^{n-1}$ possible circlings which keeps $x_{m}$, say, uncircled, and then sum up with all their possible counterparts which have $x_{m}$ circled, then the result is zero. But this is just the sum of all possible circlings of $F$,

$$
\sum_{\text {circlings }} F\left(x_{1}, x_{2}, \ldots, x_{n}\right)=0 .
$$

This is the so-called largest time equation. Notice that in a theory containing unstable ghosts Eq. (80) can only be found by using the largest time of the stable particles.

\section{The cutting equation}

Observe that the energy conservation in each vertex together with the energy flow dictated by the theta functions appearing in the cut propagators imply that many diagrams in Eq. (80) will be zero. In fact, the only

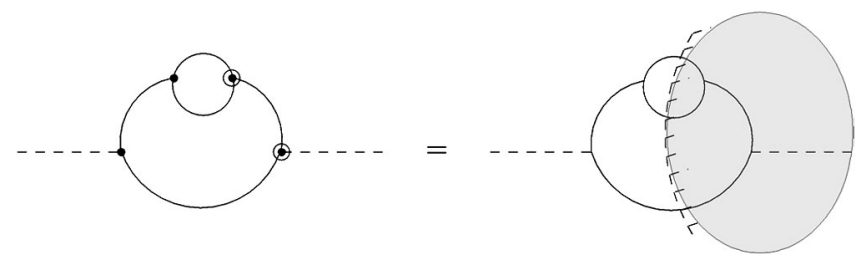

FIG. 9. A pictorial representation of a diagram with three cut lines. Dashed lines represent incoming and/or outgoing stable particles.

nonvanishing diagrams are the ones in which circled vertices form connected regions that comprise one or more outgoing lines. For such nonvanishing diagrams, one is allowed to drop the circles and mark such regions using a boundary line, which intersects one or more lines connecting uncircled and circled vertices. A typical example is illustrated in Fig. 9. The circling rules quoted above implies that such cut lines are represented by cut propagators $G^{+}$. Moreover, inside such connected regions (now represented by shaded parts in the diagram), all lines that were not cut should be represented by the complex conjugate of the propagator since they joined two circled vertices. In addition, all propagators associated with external lines connected to the diagram under consideration inside the shaded region should also be complex conjugated. Vertices inside such regions also carry an overall minus sign on the account of the circling rules. Notice that the shaded region in Fig. 9 satisfies a similar rule as compared to the shaded regions drawn in Figs. 4-6 in the sense that the propagators inside them are complex conjugated.

In this procedure, only the fully uncircled diagram [denoted by $F\left(x_{1}, x_{2}, \ldots, x_{n}\right)$ ] and the fully circled diagram [denoted by $\left.\bar{F}\left(x_{1}, x_{2}, \ldots, x_{n}\right)\right]$ can both be drawn without a cut since they will not contain any cut propagators. Equation (80) can thus be rewritten as

$$
\begin{gathered}
F\left(x_{1}, x_{2}, \ldots, x_{n}\right)+\bar{F}\left(x_{1}, x_{2}, \ldots, x_{n}\right) \\
=-\sum_{\text {cuttings }} F\left(x_{1}, x_{2}, \ldots, x_{n}\right) .
\end{gathered}
$$

This equation is also known as the cutting equation, or Cutkosky's cutting rule. Observe that energy is forced to flow toward the shaded region.

\section{E. Proof of unitarity to all orders}

Now we would like to compare Eqs. (62) and (81). First let us consider the left-hand sides of such equations. On the account of the circling rules, note that $\bar{F}$ is obtained from $F$ by complex conjugation of all propagators and multiplication by -1 of each vertex. In momentum space, this means that one must consider the replacement of every propagator with its complex conjugate in addition to reversing the direction of its momentum, $G(k) \rightarrow G^{*}(-k)$. In general, the usual Feynman rules attach an imaginary unit to 
each vertex (times possibly a coupling constant). From the third circling rule quoted above, one finds that every vertex in $\bar{F}$ should be complex conjugated if the coupling constants are real. The latter requirement is fulfilled if the Hamiltonian is Hermitian and contains only real fields. All such considerations imply that if $F$ is a diagram contributing to the process $a \rightarrow b$, then $\bar{F}$ describes the complex conjugate of the reversed process. In other words,

$$
\begin{aligned}
& \sum_{\text {diagrams }}\left[F_{a \rightarrow b}\left(k_{1}, k_{2}, \ldots, k_{n}\right)+\bar{F}_{a \rightarrow b}\left(k_{1}, k_{2}, \ldots, k_{n}\right)\right] \\
& \quad=\sum_{\text {diagrams }}\left[F_{a \rightarrow b}\left(k_{1}, k_{2}, \ldots, k_{n}\right)+F_{b \rightarrow a}^{*}\left(k_{1}, k_{2}, \ldots, k_{n}\right)\right] \\
& \quad=i \mathcal{M}(a \rightarrow b)-i \mathcal{M}^{*}(b \rightarrow a)
\end{aligned}
$$

where we summed over all possible diagrams contributing to the process $a \rightarrow b$, and $F\left(k_{1}, k_{2}, \ldots, k_{n}\right)$ and $\bar{F}\left(k_{1}\right.$, $\left.k_{2}, \ldots, k_{n}\right)$ are the Fourier transforms of $F\left(x_{1}, x_{2}, \ldots, x_{n}\right)$ and $\bar{F}\left(x_{1}, x_{2}, \ldots, x_{n}\right)$, respectively. On the other hand, if there are any complex fields, then the coupling constants can be complex. In this case, one should also consider the complex conjugate of each interaction term, if one assumes that the Hamiltonian should be Hermitian. Again, the circling rules will imply that $\bar{F}$ represents the complex conjugate of reversed processes [35].

Now let us compare the right-hand sides of Eqs. (62) and (81). Following Ref. [35,36], first consider the case in which only one internal line is being cut (we do not specify in particular whether such a line is associated with a stable or unstable particle). In this case, the diagram can be written as, in momentum space

$$
\begin{aligned}
F_{\text {single cut }}(a \rightarrow b)= & F_{\text {left }}(a \rightarrow k) G^{+}\left(k^{2}\right) \\
& \times \bar{F}_{\text {right }}(k \rightarrow b)(2 \pi)^{4} \delta^{4}(a-b),
\end{aligned}
$$

where $a$ collectively represents the sum of the momenta associated with the initial states and $b$ that of the final states. In addition, $G^{+}$represents generically a cut propagator, not necessarily associated with a specific kind of particle (stable or unstable). In particular, for the unstable ghost, one also has an overall minus sign in the expression for $G^{+}$; see Eq. (78). Notice that $\bar{F}_{\text {right }}$ corresponds to the part of the diagram inside the shaded region, and hence it implies that all vertices in the region to the right of the cut are circled. The momentum $k$ of the cut line is equal to the total incoming momenta and outgoing momenta.

The diagrams $F_{\text {left }}(a \rightarrow k)$ and $\bar{F}_{\text {right }}(k \rightarrow b)$ cannot represent contributions to scattering matrix elements as they stand due to two main reasons:

(1) The absence of the Lorentz-invariant phase space.

(2) The momentum $k$ is not on shell.

By rewriting $G^{+}\left(k^{2}\right)$ as

$$
\begin{aligned}
G^{+}\left(k^{2}\right) & =2 \pi \theta\left(k_{0}\right) \int_{0}^{\infty} d s \delta\left(k^{2}-s\right) \frac{\sigma(s)}{\pi}=\int_{0}^{\infty} d s \frac{\sigma(s)}{\pi} \int \frac{d^{4} q}{(2 \pi)^{3}} \theta\left(q_{0}\right) \delta\left(q^{2}-s\right)(2 \pi)^{4} \delta^{4}(q-k) \\
& =\left.\int_{0}^{\infty} d s \frac{\sigma(s)}{\pi} \int \frac{d^{3} q}{(2 \pi)^{3}} \frac{1}{2 \sqrt{\mathbf{q}^{2}+s}}(2 \pi)^{4} \delta^{4}(q-k)\right|_{q^{0}=\sqrt{\mathbf{q}^{2}+s}},
\end{aligned}
$$

one obtains that

$$
\begin{aligned}
F_{\text {single cut }}(a \rightarrow b)= & \int_{0}^{\infty} d s \frac{\sigma(s)}{\pi} \int \frac{d^{3} q}{(2 \pi)^{3}} \frac{1}{2 \sqrt{\mathbf{q}^{2}+s}}\left[F_{\text {right }}^{*}(b \rightarrow q) F_{\text {left }}(a \rightarrow q)\right. \\
& \left.\times(2 \pi)^{4} \delta^{4}(a-q)\right]\left.\right|_{q^{0}=\sqrt{\mathbf{q}^{2}+s}}(2 \pi)^{4} \delta^{4}(a-b) .
\end{aligned}
$$

The generalization to multiple cuts is straightforward [35,36] (cf. Fig. 9),

$$
\begin{aligned}
F_{\text {cut }}(a \rightarrow b)= & \left(\prod_{i} \int_{0}^{\infty} d s_{i} \frac{\sigma\left(s_{i}\right)}{\pi} \int \frac{d^{3} q_{i}}{(2 \pi)^{3}} \frac{1}{2 \sqrt{\mathbf{q}_{i}^{2}+s_{i}}}\right)\left[F_{\text {right }}^{*}\left(b \rightarrow\left\{q_{i}\right\}\right) F_{\text {left }}\left(a \rightarrow\left\{q_{i}\right\}\right)\right. \\
& \left.\times(2 \pi)^{4} \delta^{4}\left(a-\sum_{i} q_{i}\right)\right]\left.\right|_{q_{i}^{0}=\sqrt{\mathbf{q}_{i}^{2}+s}}(2 \pi)^{4} \delta^{4}(a-b) .
\end{aligned}
$$

We remark that here $\sigma(s)$ should be envisaged as representing the spectral function for any kind of particle, stable or unstable. 
So we see that the Lorentz-invariant phase space emerges, but $q$ is not on shell. This will only happen if the spectral function $\sigma(s)$ has a contribution from oneparticle states. Otherwise, $F_{\text {cut }}$ will not represent a contribution to the imaginary part of the scattering amplitude and hence will not contribute to the right-hand side of Eq. (62). From the Lehmann representations described above, we notice that this is the case only for stable particles. Unstable particles of any type, including the ghostlike resonances that appear in Lee-Wick models and quadratic gravity, will not contribute to unitarity sums. Unitarity is satisfied by the inclusion of only cuts from stable states in the unitarity sum. Finally, observe that the sum over all possible cuttings is identical to the sum over all possible sets of intermediate states.

The fact that only stable states should enter in the unitarity sum can also be seen from a simple usage of the LSZ reduction formula [48]

$$
\langle b|S| a\rangle=\left[i \int d^{4} x_{1} e^{-i p_{1} \cdot x_{1}}\left(\square_{1}+m^{2}\right)\right] \cdots\left[i \int d^{4} x_{n} e^{i p_{n} \cdot x_{n}}\left(\square_{n}+m^{2}\right)\right]\left\langle\Omega\left|T\left\{\phi\left(x_{1}\right) \cdots \phi\left(x_{n}\right)\right\}\right| \Omega\right\rangle
$$

associated with a quantum field $\phi(x)$ with mass $m$ and vacuum state $|\Omega\rangle$ of the full theory. By writing $S=1+i T$ and $\langle b|i T| a\rangle=(2 \pi)^{4} \delta^{4}\left(\sum p\right) i \mathcal{M}$, one observes that the matrix element $i \mathcal{M}$ can be formally deduced from Eq. (87). The exact $n$-point function will have the general structure [48]

$$
\left\langle\Omega\left|T\left\{\phi\left(x_{1}\right) \cdots \phi\left(x_{n}\right)\right\}\right| \Omega\right\rangle=\int \frac{d^{4} q_{1}}{(2 \pi)^{4}} e^{i q_{1} \cdot x_{1}} \cdots \int \frac{d^{4} q_{n}}{(2 \pi)^{4}} e^{-i q_{n} \cdot x_{n}} i G\left(q_{1}^{2}\right) \cdots i G\left(q_{n}^{2}\right) \times \mathcal{D}\left(q_{1}, q_{2}, \ldots, q_{n}\right)(2 \pi)^{4} \delta^{4}\left(\sum_{i} q_{i}\right),
$$

where $\mathcal{D}\left(q_{1}, q_{2}, \ldots, q_{n}\right)$ represents the sum of all amputated $n$-point diagrams. The exact propagators $G\left(q_{j}^{2}\right)$ depicting the external legs can be written in terms of the Lehmann representations discussed above. On the other hand, the sum in Eq. (62) is over all possible sets of intermediate states, which may include states associated with unstable particles. However, when we use Eq. (87) to calculate, say, the $S$-matrix elements $\langle f|S| a\rangle$ and then extract from it the associated scattering amplitude $\mathcal{M}(a \rightarrow f)$, one will get a nonvanishing contribution only if the propagators $G\left(q_{f}^{2}\right)$ have a term associated with one-particle states. In particular, this implies that the right-hand side of Eq. (62) will not contain any contribution from intermediate unstable states.

There is one point that we have not discussed so far and that deserves a careful attention. All the above derivations were carried out for scalar fields. Does such a procedure generalize to more general fields carrying additional degrees of freedom? We know that, for the generalized optical theorem to hold in general, the numerator of a propagator must be equal to the sum over physical spin states [48]. So, this sum must also be present in the numerator of the cut propagators in order to identify the right-hand sides of Eqs. (62) and (81). This is indeed the case for fermions, and one can easily decompose the fermionic propagator into positive- and negative-frequency parts [34]. A Lehmann representation is also available [52,53]. On the other hand, for photons the argument is more subtle. Indeed, the numerator of the propagator is not just the sum over physical polarizations [48]: both have a distinct longitudinal part. But also both carry a $\eta_{\mu \nu}$ factor. So, using the Ward identity for the scattering amplitudes (which contain a sum over physical polarizations) and gauge invariance for the propagator (which allows us to eliminate the longitudinal term by choosing, say, the Feynman gauge), then it is easy to show that unitarity holds. In particular, in the Feynman gauge, the photon propagator can be decomposed into positive- and negativefrequency parts [35]. A Lehmann representation can also be derived for the photon propagator [52,53]. In turn, as above a full spectral representation for the associated ghostlike propagator can be easily obtained from the modified Lehmann representation in the Feynman gauge [51]. The Ward identity in Lee-Wick gauge theory has also been demonstrated [54].

As one can note from the above discussion, the case of quadratic gravity deserves a careful treatment. Details concerning the Ward identity and the sum over physical spin states should be taken into account. Moreover, when considering an explicit expression for the spectral function, one generally considers a resummation for internal propagators and then extracts the imaginary part of the resulting expression. But it is known that such a resummation procedure may violate the Ward identity if not performed with due care [35]. Hence, one must be careful in generalizing the procedure adopted here for the case of quadratic gravity. We hope to fully explore this issue in a future work.

\section{THE NARROW-WIDTH APPROXIMATION AND THE LEE-WICK CONTOUR}

Although the unitarity prescription is to include only cuts on the stable particles and not on the resonances, in practice we often ignore this when the resonances live long enough 
that we can treat them as stable in practical calculations. For normal resonances, this narrow width approximation (NWA) yields the correct answer in the limit that the width can be neglected. In this section, we treat ghostlike resonances in this limit. We will see that we get a correct result if we take the $\Gamma \rightarrow 0$ limit in the proper calculation, with stable particle cuts. However, if we were to treat the ghost as a stable particle from the start, we would get a different and incorrect answer. This can be rescued by a slight change of the integration contour, that proposed by Lee and Wick.

Here is how the NWA works. When studying loops with the resonance inside the loop, we can calculate the cuts using the usual cutting rules on the stable particles. We did this explicitly for two- and three-particle cuts in Sec. IV. As seen in Eq. (54), this yields

$\operatorname{Disc} D(q)=D(q) 2 q \Gamma(q) D^{*}(q)=\frac{2 q \Gamma(q)}{\left(q^{2}-m_{r}^{2}\right)^{2}+\left(m_{r} \Gamma(q)\right)^{2}}$

by the unitarity relation for the self-energy. In the limit that the width goes to zero, the contribution becomes vanishingly small except very close to the resonance. Using the representation of the delta function

$$
\delta(x)=\lim _{\epsilon \rightarrow 0} \frac{1}{\pi} \frac{\epsilon}{x^{2}+\epsilon^{2}},
$$

one finds that

$$
\lim _{\Gamma \rightarrow 0} \operatorname{Disc} D(q)=2 \pi \delta\left(q^{2}-m_{r}^{2}\right),
$$

which reproduces the cutting rule for a stable particle. We note that, following from the work that we have presented above, this result is true for both normal resonances and ghost resonances. This implies that the usual cutting rules also apply to ghosts if we wish to approximate them as stable particles.

However, if one starts with the ghost field in a loop and calculates naively, one does not get the correct result. This can be demonstrated by exploring the three-particle cut, which we calculated explicitly above. In this case, the NWA for the process consists of one massless field with the usual $i \epsilon$ structure plus one massive ghost field with a small width. Because the ghost is unstable and decays to two massless fields, the actual intermediate state will be three massless fields. We will show that this cut naively vanishes when calculated in the standard manner. ${ }^{3}$

Consider the amplitude with one massless field and one ghost

\footnotetext{
${ }^{3}$ There are many ways to obtain this result. Our presentation closely follows that of Schwartz [48], and the reader can see there how the cutting rules arise for nonghost fields.
}

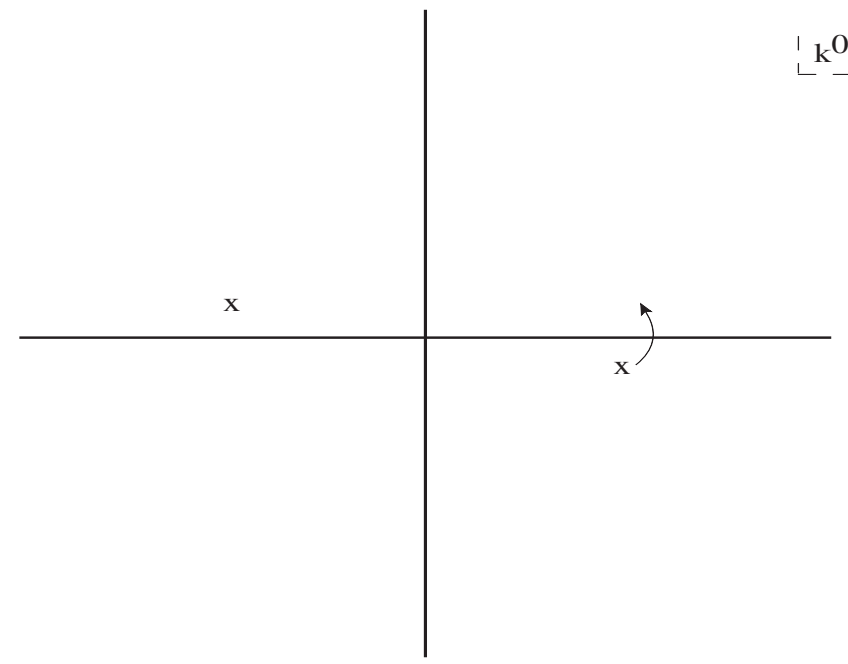

FIG. 10. The shift in pole location relating the Feynman propagator and the advanced propagator.

$$
i \mathcal{M}=-\int \frac{d^{4} k}{(2 \pi)^{4}} \frac{i}{(k-q)^{2}+i \epsilon} \frac{-i}{k^{2}-m^{2}-i \gamma} .
$$

We will treat the width $\gamma$ as a small parameter. By shifting the poles in the normal Feynman propagator as illustrated in Fig. 10, one write it in terms of the advanced propagator with poles only above the real axis via

$$
\frac{i}{k^{2}+i \epsilon}=i D_{A}(k)+\frac{\pi}{\omega_{k}} \delta\left(k_{0}-\omega_{k}\right)
$$

with $\omega_{k}=|\mathbf{k}|$, and

$$
D_{A}=\frac{1}{2 \omega_{k}}\left(\frac{1}{k_{0}-\omega_{k}-i \epsilon}-\frac{1}{k_{0}+\omega_{k}-i \epsilon}\right)
$$

Using the pole structure shown in Sec. III, one can do the equivalent transformation for the ghost propagator

$$
\frac{-i}{k^{2}-m^{2}-i \gamma}=-i D \text { "A" }(k)+\frac{\pi}{E_{k}} \delta\left(k_{0}+E_{k-p}\right),
$$

with $E_{k}=\sqrt{\mathbf{k}^{2}+\mathbf{m}^{2}}$, valid for infinitessimal $\gamma$, with

$$
D \text { "A" }(k)=\frac{1}{2 E_{k}}\left(\frac{1}{k_{0}-E_{k}-i \gamma}-\frac{1}{k_{0}+E_{k}-i \gamma}\right)
$$

using the shift of poles illustrated in Fig. 11. Here we have put the $A$ in quotes as one notes from the work in Sec. III that this is not the actual ghost propagator with advanced boundary conditions. However, it has poles only above the real axis, and that is what is important in the present calculation. 


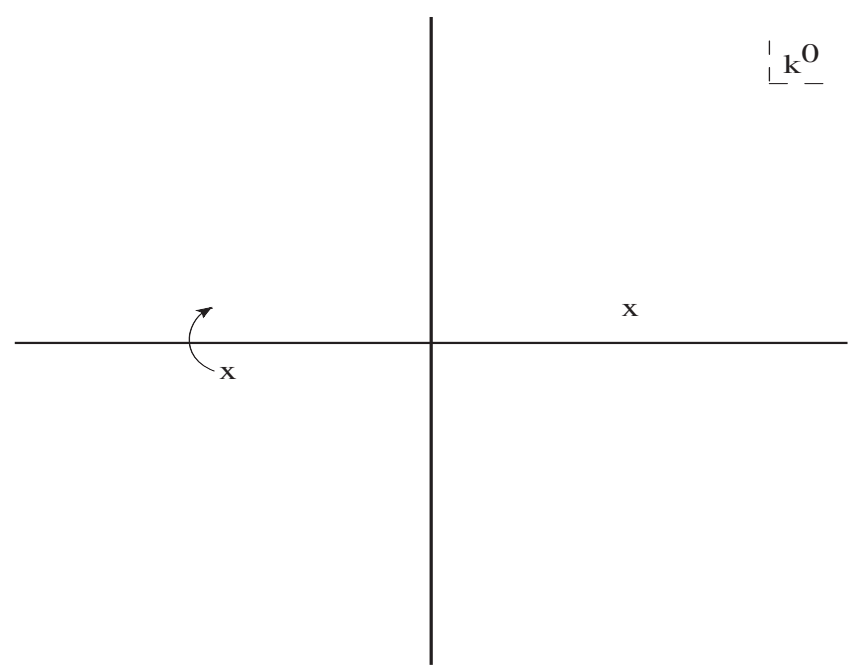

FIG. 11. The shift of a pole for the ghost particle.

The amplitude then turns into

$$
\begin{aligned}
i \mathcal{M}= & -\int \frac{d^{4} k}{(2 \pi)^{4}}\left[i D_{A}(k-q)+\frac{\pi}{\omega_{k-q}} \delta\left(k_{0}-q_{0}-\omega_{k-q}\right)\right] \\
& \times\left[-i D \text { "A" }(k)+\frac{\pi}{E_{k}} \delta\left(k_{0}+E_{k}\right)\right] .
\end{aligned}
$$

If we work in the center of mass where $q$ is purely timelike, then $\omega_{k-q}=\omega_{k}=|\mathbf{k}|$. The contour can be closed below the real axis for the product of the two advanced propagators, with a vanishing result. The two delta functions cannot be simultaneously satisfied. Only the cross-terms remain.

Having discarded the vanishing terms, the advanced propagators can be converted back into Feynman propagators, such that

$$
\begin{aligned}
\mathcal{M}= & \int \frac{d^{4} k}{(2 \pi)^{4}}\left[D_{F}(k-q) \frac{\pi}{E_{k}} \delta\left(k_{0}+E_{k}\right)\right. \\
& \left.+D_{F}^{g}(k) \frac{\pi}{\omega_{k}} \delta\left(k_{0}-q_{0}-\omega_{k}\right)\right] .
\end{aligned}
$$

Finally, we can pick out the imaginary part of this amplitude

$$
\begin{aligned}
\operatorname{Im}[\mathcal{M}]= & -\int \frac{d^{4} k}{(2 \pi)^{4}}\left[\pi \delta\left((k-q)^{2}\right) \frac{\pi}{E_{k}} \delta\left(k_{0}+E_{k}\right)\right. \\
& \left.-\frac{\pi}{\omega_{k}} \delta\left(k_{0}-q_{0}-\omega_{k}\right) \pi \delta\left(k^{2}-m^{2}\right)\right] .
\end{aligned}
$$

In both cases, the delta functions cannot be simultaneously satisfied. In the first term, we have $k_{0}=-E_{k}$, while the second one would then require $\left(-\sqrt{\vec{k}^{2}+m^{2}}-q_{0}\right)^{2}=\vec{k}^{2}$, which is impossible. The second term also vanishes because $q_{0}>m$. Calculated this way, the imaginary part

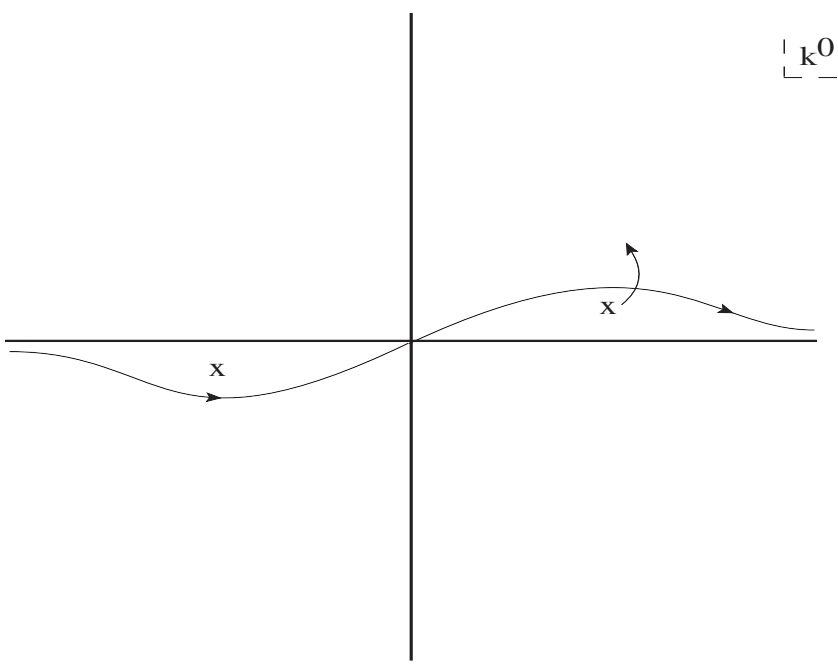

FIG. 12. Using the Lee-Wick contour.

of the amplitude vanishes, in contrast with the direct calculation in Eq. (61).

We note that the problem comes from the $\delta\left(k_{0}+E_{k}\right)$ in the first term of this expression, which can be traced back to the same factor in Eq. (95). For a normal particle, this factor is $\delta\left(k_{0}-E_{k}\right)$, in which case both delta functions can be satisfied, yielding the usual rule for the cut. This problem is solved by a modification of the contour in doing the loop momentum integration, as originally described by Lee and Wick. The contour is chosen to run above the positive pole and below the negative one, as illustrated in Fig. 12. Using this contour, the shift in the pole location will now yield a factor of $\delta\left(k_{0}-E_{k}\right)$, and we get the usual cutting rule, as required.

This modification of the contour is introduced in order to reproduce the result of the correct discontinuity calculation presented in Sec. IV. It is needed because one has attempted to (improperly) take the cut across the unstable ghost as if it were a stable particle.

\section{SUMMARY}

The ghostlike modes which occur in theories with quartic propagators require a re-evaluation of many of the basic properties of quantum field theory. A crucial observation is that these modes correspond to an unstable resonance, because of their coupling to lighter states in the theory. There are important consequences of this fact. This removes these modes from the asymptotic spectrum, which consists only of stable particle states. In a path integral treatment, one does not have to perform free-field quantization for such states as they appear only in intermediate state propagators. We have shown that the energy flow is different than normal particles: what is usually claimed as positive energy propagates backwards in time. Nevertheless, the various Green functions imply stability in Minkowski space. The propagation in time is backward from normal particles, and this 
contributes to a violation of microcausality over timescales of order the inverse width of these modes. ${ }^{4}$

Most importantly, building on the original work by Lee and Wick, we have given a proof that unitarity is satisfied to all orders in such theories and provided explicit examples of how this occurs. Discontinuities are calculated by only including cuts on the stable particles of the theory. The proof follows the technique of Veltman, which was originally applied to reach the same conclusion about normal resonances. However, the narrow-width approximation, in which cuts are applied to the unstable particles as if they were stable, appears differently for these resonances, and this can be accounted for in this limit by using the LeeWick contour. It should be understood that the Lee-Wick contour must be used only when one allows for the ghost as a particle in a loop (and this can only be done in the narrowwidth approximation). Otherwise, the standard contour for the Feynman propagator is to be used.

An important point that should be highly appreciated is that the stable particle and the resonance appear in the same propagator, which means that eventually we are discussing two features of a single (quartic) propagator. The separation into two propagators (or the introduction of an auxiliary field at the level of the action) is to some extent artificial and it should be envisaged only as a convenient procedure

${ }^{4}$ We discuss causality further in a separate paper [31]. in order to unveil in a manifest way the ghost feature of the propagator.

Overall, we see that these higher derivative theories are healthier than originally expected, as long as the microcausality violation occurs over short enough time scales that it is unconstrained by present experiments. While one can make higher derivative variants of any theory, the most important physical application is to gravity. The gravitational interaction requires higher derivatives in the action for the renormalizaton of the theory, and indeed quadratic gravity proves to be a renormalizable theory of quantum gravity. It follows from our work here that it is also unitary and stable near flat space. It is in this regard the most conservative ultraviolet completion of quantum gravity and deserves further exploration.

\section{ACKNOWLEDGMENTS}

We would like to thank the following for useful comments or discussions: C. Burgess, A. Denner, B. Holdom, B. Holstein, P. Mannheim, D. O'Connell, M. Peskin, J. Ren, A. Salvio, M. Schwartz, G. 't Hooft, A. Tolley, and C. Wetterich. The work of J.F.D. has been partially supported by the U.S. National Science Foundation under Grant No. NSF-PHY18-20675. The work of G. M. has been partially supported by Conselho Nacional de Desenvolvimento Científico e Tecnológico- $\mathrm{CNPq}$ under Grant No. 310291/2018-6 and Fundação Carlos Chagas Filho de Amparo à Pesquisa do Estado do Rio de JaneiroFAPERJ under Grant No. E-26/202.725/2018.
[1] K. S. Stelle, Renormalization of higher derivative quantum gravity, Phys. Rev. D 16, 953 (1977).

[2] A. Salam and J. A. Strathdee, Remarks on high-energy stability and renormalizability of gravity theory, Phys. Rev. D 18, 4480 (1978).

[3] J. Julve and M. Tonin, Quantum gravity with higher derivative terms, Nuovo Cimento B 46, 137 (1978).

[4] E. S. Fradkin and A. A. Tseytlin, Renormalizable asymptotically free quantum theory of gravity, Phys. Lett. 104B, 377 (1981); E. S. Fradkin and A. A. Tseytlin, Renormalizable asymptotically free quantum theory of gravity, Nucl. Phys. B201, 469 (1982).

[5] E. T. Tomboulis, Renormalization and unitarity in higher derivative and nonlocal gravity theories, Mod. Phys. Lett. A 30, 1540005 (2015); E. T. Tomboulis, 1/N expansion and renormalization in quantum gravity, Phys. Lett. 70B, 361 (1977); I. Antoniadis and E. T. Tomboulis, Gauge invariance and unitarity in higher derivative quantum gravity, Phys. Rev. D 33, 2756 (1986).

[6] D. A. Johnston, Sedentary ghost poles in higher derivative gravity, Nucl. Phys. B297, 721 (1988).
[7] A. Salvio, Quadratic gravity, Front. Phys. 6, 77 (2018).

[8] J. F. Donoghue and G. Menezes, Gauge assisted quadratic gravity: A framework for UV complete quantum gravity, Phys. Rev. D 97, 126005 (2018).

[9] M. B. Einhorn and D. R. T. Jones, Naturalness and dimensional transmutation in classically scale-invariant gravity, J. High Energy Phys. 03 (2015) 047; T. Jones and M. Einhorn, Quantum gravity and dimensional transmutation, Proc. Sci., PLANCK2015 (2015) 061.

[10] A. Salvio and A. Strumia, Agravity, J. High Energy Phys. 06 (2014) 080; Agravity up to infinite energy, Eur. Phys. J. C 78, 124 (2018).

[11] J.F. Donoghue, Quartic propagators, negative norms and the physical spectrum, Phys. Rev. D 96, 044007 (2017).

[12] J.F. Donoghue and G. Menezes, Inducing the Einstein action in QCD-like theories, Phys. Rev. D 97, 056022 (2018).

[13] B. Holdom and J. Ren, QCD analogy for quantum gravity, Phys. Rev. D 93, 124030 (2016); Quadratic gravity: From weak to strong, Int. J. Mod. Phys. D 25, 1643004 (2016). 
[14] P. D. Mannheim, Unitarity of loop diagrams for the ghostlike $1 /\left(k^{2}-M_{1}^{2}\right)-1 /\left(k^{2}-M_{2}^{2}\right)$ propagator, Phys. Rev. D 98, 045014 (2018); Making the case for conformal gravity, Found. Phys. 42, 388 (2012).

[15] G. 't Hooft, The conformal constraint in canonical quantum gravity, arXiv:1011.0061; Local conformal symmetry: The missing symmetry component for space and time, Int. J. Mod. Phys. D 24, 1543001 (2015); Singularities, horizons, firewalls, and local conformal symmetry, Springer Proc. Phys. 208, 1 (2018).

[16] S. D. Odintsov and I. L. Shapiro, General relativity as the low-energy limit in higher derivative quantum gravity, Classical Quantum Gravity 9, 873 (1992); Teor. Mat. Fiz. 90, 469 (1992) [Curvature phase transition in $R^{2}$ quantum gravity and induction of Einstein gravity, Theor. Math. Phys. 90, 319 (1992)]; I. L. Shapiro and G. Cognola, Interaction of low-energy induced gravity with quantized matter and phase transition induced to curvature, Phys. Rev. D 51, 2775 (1995); I. L. Shapiro, Hilbert-Einstein action from induced gravity coupled with scalar field, Mod. Phys. Lett. A 09, 1985 (1994).

[17] A. V. Smilga, Ghost-free higher-derivative theory, Phys. Lett. B 632, 433 (2006); Classical and quantum dynamics of higher-derivative systems, Int. J. Mod. Phys. A 32, 1730025 (2017).

[18] G. Narain and R. Anishetty, Short distance freedom of quantum gravity, Phys. Lett. B 711, 128 (2012); G. Narain, Signs, and stability in higher-derivative gravity, Int. J. Mod. Phys. A 33, 1850031 (2018); G. Narain and R. Anishetty, Unitary and renormalizable theory of higher derivative gravity, J. Phys. Conf. Ser. 405, 012024 (2012).

[19] D. Anselmi, On the quantum field theory of the gravitational interactions, J. High Energy Phys. 06 (2017) 086; Fakeons and Lee-Wick models, J. High Energy Phys. 02 (2018) 141; D. Anselmi and M. Piva, Perturbative unitarity of Lee-Wick quantum field theory, Phys. Rev. D 96, 045009 (2017).

[20] I. L. Buchbinder, S. D. Odintsov, and I. L. Shapiro, Effective Action in Quantum Gravity (IOP, Bristol, United Kingdom, 1992).

[21] L. Alvarez-Gaume, A. Kehagias, C. Kounnas, D. Lüst, and A. Riotto, Aspects of quadratic gravity, Fortschr. Phys. 64, 176 (2016).

[22] T. D. Lee and G. C. Wick, Negative metric and the unitarity of the S matrix, Nucl. Phys. B9, 209 (1969).

[23] T. D. Lee and G. C. Wick, Unitarity in the $N \theta \theta$ sector of soluble model with indefinite metric, Nucl. Phys. B10, 1 (1969).

[24] T.D. Lee and G.C. Wick, Finite theory of quantum electrodynamics, Phys. Rev. D 2, 1033 (1970).

[25] R. E. Cutkosky, P. V. Landshoff, D. I. Olive, and J. C. Polkinghorne, A non-analytic S matrix, Nucl. Phys. B12, 281 (1969).

[26] S. Coleman, Acausality, in Erice 1969: Ettore Majorana Schoool on Subnuclear Phenomena, edited by A Zicchici (Academic Press, New York, 1970), p. 282.

[27] B. Grinstein, D. O'Connell, and M. B. Wise, The Lee-Wick standard model, Phys. Rev. D 77, 025012 (2008).

[28] B. Grinstein, D. O'Connell, and M. B. Wise, Causality as an emergent macroscopic phenomenon: The Lee-Wick O(N) model, Phys. Rev. D 79, 105019 (2009).
[29] L. Modesto and I. L. Shapiro, Superrenormalizable quantum gravity with complex ghosts, Phys. Lett. B 755, 279 (2016).

[30] A. Accioly, B. L. Giacchini, and I. L. Shapiro, Lowenergy effects in a higher-derivative gravity model with real and complex massive poles, Phys. Rev. D 96, 104004 (2017).

[31] J.F. Donoghue and G. Menezes, Arrow of Causality and Quantum Gravity, Phys. Rev. Lett. 123, 171601 (2019).

[32] R. E. Cutkosky, Singularities and discontinuities of Feynman amplitudes, J. Math. Phys. (N.Y.) 1, 429 (1960).

[33] M. Veltman, Unitarity and causality in a renormalizable field theory with unstable particles, Physica (Utrecht) 29, 186 (1963).

[34] G. 't Hooft and M. J. G. Veltman, Diagrammar, in Particle Interactions at Very High Energies, edited by D. Speiser, F. Halzen, and J. Weyers, NATO Advanced Study Institutes, Series B, Vol. 4 (Plenum Press, New York, 1974), p. 177

[35] J. Rodenburg, Unstable particles and resonances, Master thesis, Utrecht University, 2015.

[36] J.-N. O. Lang, The complex mass scheme, gauge dependence and unitarity in perturbative quantum field theory, Master thesis, Wurzburg University, 2013.

[37] A. Denner and J. N. Lang, The complex-mass scheme and unitarity in perturbative quantum field theory, Eur. Phys. J. C 75, 377 (2015).

[38] C. M. Bender and P. D. Mannheim, No-Ghost Theorem for the Fourth-Order Derivative Pais-Uhlenbeck Oscillator Model, Phys. Rev. Lett. 100, 110402 (2008).

[39] A. Salvio and A. Strumia, Quantum mechanics of 4-derivative theories, Eur. Phys. J. C 76, 227 (2016).

[40] M. Raidal and H. Veerme, On the quantisation of complex higher derivative theories and avoiding the ostrogradsky ghost, Nucl. Phys. B916, 607 (2017).

[41] S. C.d. Reis, G. Chapiro, and I. L. Shapiro, Beyond the linear analysis of stability in higher derivative gravity with the Bianchi-I metric, Phys. Rev. D 100, 066004 (2019).

[42] A. Salvio, Metastability in quadratic gravity, Phys. Rev. D 99, 103507 (2019).

[43] J. F. Donoghue and B. K. El-Menoufi, Nonlocal quantum effects in cosmology: Quantum memory, nonlocal FLRW equations, and singularity avoidance, Phys. Rev. D 89, 104062 (2014).

[44] N. D. Birrell and P. C. W. Davies, Quantum Fields in Curved Space (Cambridge University Press, Cambridge, United Kingdom, 1982).

[45] L. Parker and D. Toms, Quantum Field Theory in Curved Spacetime (Cambridge University Press, Cambridge, United Kingdom, 2009).

[46] J. F. Donoghue and B. K. El-Menoufi, QED trace anomaly, non-local Lagrangians and quantum equivalence principle violations, J. High Energy Phys. 05 (2015) 118.

[47] J. F. Donoghue and B. K. El-Menoufi, Covariant non-local action for massless QED and the curvature expansion, J. High Energy Phys. 10 (2015) 044. 
[48] M. D. Schwartz, Quantum Field Theory and the Standard Model (Cambridge University Press, Cambridge, United Kingdom, 2013).

[49] G. Kallen, On the definition of the renormalization constants in quantum electrodynamics, Helv. Phys. Acta 25, 417 (1952).

[50] H. Lehmann, On the properties of propagation functions and renormalization contants of quantized fields, Nuovo Cimento 11, 342 (1954).
[51] J. F. Donoghue and G. Menezes, Massive poles in Lee-Wick quantum field theory, Phys. Rev. D 99, 065017 (2019).

[52] S.S. Schweber, An Introduction to Relativistic Quantum Field Theory (Harper and Row, New York, 1962).

[53] C. Itzykson and J. B. Zuber, Quantum Field Theory (McGraw-Hill, NewYork, 1980).

[54] B. Grinstein, D. O'Connell, and M. B. Wise, Massive vector scattering in Lee-Wick gauge theory, Phys. Rev. D 77, 065010 (2008). 